KIAS-P01026

hep-th/0105246

\title{
Tachyon Lump Solutions of Bosonic D-branes on SU(2) Group Manifolds in Cubic String Field Theory
}

\author{
Yoji Michishita * \\ School of Physics, Korea Institute for Advanced Study \\ 207-43, Cheongryangri, Dongdaemun, Seoul, 130-012, Korea
}

May, 2001

\begin{abstract}
We construct tachyon lump solutions of bosonic D2-branes on $\mathrm{SU}(2)$ group manifolds by level truncation approximation in cubic string field theory, which are regarded as D0-branes. The energies for these solutions show good agreement with the expected values.
\end{abstract}

PACS codes: 11.25.-w, 11.25.Sq

Keywords: string field theory, D-brane, tachyon condensation

*michishi@kias.re.kr 


\section{Introduction}

Tachyon condensation on D-branes has been studied extensively. It is conjectured for bosonic D-branes that the minimum of the tachyon potential represents closed string vacuum [1], and tachyon lump solutions represent lower dimensional branes [1, 2].

String field theories are powerful tools for checking these conjectures. In many cases one of the following two types of string field theories has been used : cubic string field theory (CSFT) [3] and boundary string field theory (BSFT) [4. By level truncation approximation in CSFT we can calculate numerically the value of the potential at the minimum [5, 6], construct tachyon lump solutions [0], and confirm the absence of physical excitation on the vacuum [8]. Furthermore, the form of the action around the closed string vacuum (vacuum SFT) is conjectured in [9] and its properties are investigated in [10, 11]. On the other hand, by using BSFT we can get the exact tachyon potential and lump solutions [12]. (For related works and the case of superstring see [13, 14, 15]. For a review see [16].) At present the relation between these two string field theories is unclear. Therefore it is worth investigating the tachyon condensation by both of them. In this paper we use CSFT and level truncation approximation.

In CSFT it is proven that the tachyon potential can be determined irrelevantly to the choice of closed string background [17]. The crucial point for the proof of this fact is that we can drop all the fields except the unit operator and its descendants. However, for lump solutions we must give nonzero vev to nontrivial primary operators and their descendants, and therefore the results depend on the choice of closed string background. The solutions constructed in [7] are those on flat background.

For further understanding of tachyon condensation, it is desirable to consider lump solutions on curved backgrounds. In this paper we give such an example: lump solutions on SU(2) group manifold. SU(2) group manifold is an example of curved background on which the worldsheet CFT is known exactly i.e. WZW model. This manifold is also known as the example which has the noncommutative structure [18, 19]. The analyses of tachyon condensation by the worldsheet CFT and the effective action is given in [20]

D-branes which extend to two dimensions in $\mathrm{SU}(2)$ (we call them D2-branes) can be regarded as bound states of D-branes which sit at a point in $\mathrm{SU}(2)$ (we call them D0-branes) 21, 19, and D0-branes are conjectured to be able to vanish into the vacuum by tachyon condensation. Therefore one can imagine the process that some of the D0-branes forming the 
D2-brane vanish and the other D0-branes remain. Hence it is natural to conjecture that there exist lump solutions on D2-branes which represent some D0-branes.

We construct such lump solutions in CSFT in level [1,3], [2,6] and [5/2,5] approximation, where $[N, M]$ means that we consider only the fields with the level (i.e. conformal dimension+1) $<N$ and retain only the terms with the sum of the levels $<M$. (On the other hand the notation $(N, M)$ used in many literature means only the fields with the level $\leq N$ and only the terms with the sum of the levels $\leq M$.) We consider the case where the D2-brane is the bound state of 2 and 3 D0-branes.

This paper is organized as follows. In section 2, we summarize the properties of D-branes on SU(2) group manifold and the cubic string field theory on it, and explain how to calculate the terms in the action of CSFT. In section 3, we construct lump solutions and compute their energy. Section 4 contains conclusions and discussions.

In the following $\alpha^{\prime}$ is taken to be 1.

\section{D-branes and cubic string field theory on $\mathrm{SU}(2)$ group manifolds}

In this section we summarize the known results about D-branes of bosonic string theory on $\mathrm{SU}(2)$ group manifolds and fix the notations. Then we explain how to calculate the terms in the action of cubic string field theory on these manifolds.

\subsection{The worldsheet CFT}

$\mathrm{SU}(2)$ group manifold $\left(\simeq S^{3}\right)$ can be, for example, parametrized as follows.

$$
\begin{gathered}
g=\left(\begin{array}{cc}
x_{4}-i x_{2} & -x_{1}-i x_{3} \\
x_{1}-i x_{3} & x_{4}+i x_{2},
\end{array}\right) \in S U(2), \\
x_{1}=\sin \psi \sin \theta \cos \phi \\
x_{2}=\sin \psi \sin \theta \sin \phi \\
x_{3}=\sin \psi \cos \theta \\
x_{4}=\cos \psi
\end{gathered}
$$


where $\theta, \phi$ and $\psi$ are spherical coordinates of $S^{3}$. Let the radius of $S^{3}$ be $\sqrt{k \alpha^{\prime}}=\sqrt{k}$. The worldsheet CFT on SU(2) group manifold, i.e. SU(2) WZW model, has the left moving current $J^{a}(z)$ and the right moving current $\bar{J}^{a}(\bar{z})$. Their mode expansions are $J^{a}(z)=\sum_{n} z^{-n-1} J_{n}^{a}$ and similar one for $\bar{J}^{a}(\bar{z})$. The commutation relation of these modes is

$$
\left[J_{n}^{a}, J_{m}^{b}\right]=i f^{a b c} J_{n+m}^{c}+k n \delta^{a b} \delta_{n,-m}
$$

$\left(f^{a b c}=\epsilon^{a b c}\right.$ for $\left.\mathrm{SU}(2)\right)$ The radius $k$ becomes the level of the $\mathrm{SU}(2)$ current algebra. Therefore $k$ is quantized to be positive integer valued. The central charge of this CFT is $\frac{3 k}{k+2}$. $J_{0}^{a}$ form ordinary $\mathrm{SU}(2)$ algebra and primary fields belong to the representations of this algebra. We denote the primary field in spin $j$ representation by $\Phi_{j}^{m}$. Then,

$$
J_{0}^{a}\left|\Phi_{j}^{m}\right\rangle=\left|\Phi_{j}^{m^{\prime}}\right\rangle\left(T_{(j)}^{a}\right)_{m^{\prime}}^{m}, \quad J_{n>0}^{a}\left|\Phi_{j}^{m}\right\rangle=0
$$

where $\left(T_{(j)}^{a}\right)_{m^{\prime}}^{m}$ is the spin $j$ representation matrix. The conformal dimension of $\Phi_{j}^{m}$ is $h_{j} \equiv$ $\frac{j(j+1)}{k+2}$. We take the diagonal modular invariant as the closed string 1-loop partition function.

When we consider open strings we must impose some boundary condition which relates $J^{a}(z)$ to $\bar{J}^{a}(\bar{z})$. Henceforth we consider the following condition.

$$
J^{a}(z)=\bar{J}^{a}(\bar{z}) \text { on the boundary, }
$$

where $z$ is a coordinate of the upper half plane and we take the real axis as the boundary. Boundary states for this boundary condition are [23, 24]

$$
\left.|J\rangle=\sum_{j} \frac{S_{J}^{j}}{\sqrt{S_{0}^{j}}}|j\rangle\right\rangle,
$$

where $|j\rangle\rangle$ is the Ishibashi state constructed on the primary field $\Phi_{j}^{m}$ and $S_{J}^{j}$ is the modular transformation matrix:

$$
S_{J}^{j}=\sqrt{\frac{2}{k+2}} \sin \frac{(2 J+1)(2 j+1) \pi}{k+2} .
$$

$J$ takes the values $0, \frac{1}{2}, 1, \cdots, \frac{k}{2}$. The worldvolume of the boundary state labelled by $J$ is $\psi=$ const. $=\frac{(2 J+1) \pi}{k+2}$, which is an $S^{2}$ parametrized by $\theta$ and $\phi$ 22, 25]. If $J=0$ or $\frac{k}{2}$, the $S^{2}$ shrinks to a point (at $k \rightarrow \infty$ ). Thus $|J\rangle$ with $J=0, \frac{k}{2}$ represent D0-branes and others are D2-branes. The D2-brane with boundary state $|J\rangle$ can be regarded as the bound state of $(2 J+1)$ D0-branes [21, 19].

The spectrum of primary fields on the brane with boundary state $|J\rangle$ is

$$
\Phi_{j}^{m}, \quad j=0,1,2, \cdots, \min (2 J, k-2 J) .
$$


Note that $j$ takes only integers. Their OPE is known as follows [26, 18].

$\Phi_{j_{1}}^{m_{1}}(x) \Phi_{j_{1}}^{m_{1}}(y)=(x-y)^{h_{j_{3}}-h_{j_{1}}-h_{j_{2}}}\left\langle j_{1}, m_{1}, j_{2}, m_{2} \mid j_{1}, j_{2}, j_{3}, m_{3}\right\rangle c_{j_{1} j_{2}}^{j_{3}}(k, J) \Phi_{j_{3}}^{m_{3}}(y)+$ (descendants)

where $\left\langle j_{1}, m_{1}, j_{2}, m_{2} \mid j_{1}, j_{2}, j_{3}, m_{3}\right\rangle$ is the Clebsch-Gordan coefficient, and $c_{j_{1} j_{2}}^{j_{3}}(k, J)$ is written by quantum 6-j symbol and given in the appendix $\mathrm{A}$. Three point correlation functions are

$$
\left\langle\Phi_{j_{1}}^{m_{1}}\left|\Phi_{j_{2}}^{m_{2}}(1)\right| \Phi_{j_{3}}^{m_{3}}\right\rangle=C\left[\begin{array}{ccc}
m_{1} & m_{2} & m_{3} \\
j_{1} & j_{2} & j_{3}
\end{array}\right]
$$

and

$$
C\left[\begin{array}{ccc}
m_{1} & m_{2} & m_{3} \\
j_{1} & j_{2} & j_{3}
\end{array}\right]=\frac{(-1)^{m_{3}}}{\sqrt{\left(2 j_{3}+1\right)\left[2 j_{3}+1\right]}}\left\langle j_{1}, m_{1}, j_{2}, m_{2} \mid j_{1}, j_{2}, j_{3},-m_{3}\right\rangle c_{j_{1} j_{2}}^{j_{3}}(k, J)
$$

The definition of $\left[2 j_{3}+1\right]$ is given in the appendix A. $C\left[\begin{array}{ccc}m_{1} & m_{2} & m_{3} \\ j_{1} & j_{2} & j_{3}\end{array}\right]$ is symmetric under cyclic permutations of $(1,2,3)$, as should be for the three point functions, and satisfies the following relations.

$$
\begin{aligned}
C\left[\begin{array}{ccc}
m_{1} & m_{2} & m_{3} \\
j_{1} & j_{2} & j_{3}
\end{array}\right] & =0 \text { if } m_{1}+m_{2}+m_{3} \neq 0 \\
C\left[\begin{array}{ccc}
m_{1} & m_{2} & m_{3} \\
j_{1} & j_{2} & j_{3}
\end{array}\right] & =(-1)^{j_{1}+j_{2}+j_{3}} C\left[\begin{array}{ccc}
m_{2} & m_{1} & m_{3} \\
j_{2} & j_{1} & j_{3}
\end{array}\right] .
\end{aligned}
$$

\subsection{Cubic string field theory on $\mathrm{SU}(2)$}

Next we explain the cubic string field theory and how to calculate the terms in the action. We will consider the following background.

$$
X \times Y \times \mathcal{M} \times \mathrm{SU}(2),
$$

where $X$ is the time direction which is taken to be flat, and $Y$ is a spatial direction, which is also taken to be flat, and the boundary condition for open strings along this direction is taken to be Dirichlet. $\mathcal{M}$ is an arbitrary background with the central charge $24-\frac{3 k}{k+2}$, appropriate for cancelling the total central charge. We denote the Virasoro operator of the matter part of $X \times Y \times \mathcal{M} \times \mathrm{SU}(2)$ and $X \times Y \times \mathcal{M}$ by $L_{n}^{(m)}$ and $L_{n}^{\prime}$ respectively, and the central charge of $X \times Y \times \mathcal{M}$ by $c^{\prime}=26-\frac{3 k}{k+2}$

The action for string field $\Psi$ on general CFT background is constructed in [27]:

$$
S=-\frac{1}{g_{o}^{2}}\left(\frac{1}{2}\left\langle\Psi, Q_{B} \Psi\right\rangle+\frac{1}{3}\langle\Psi, \Psi, \Psi\rangle\right) .
$$


We follow the notation of [28]. $Q_{B}$ is the BRST charge:

$$
\begin{aligned}
Q_{B}= & \oint \frac{d z}{2 \pi i}: c(z)\left(T^{(m)}(z)+\frac{1}{2} T^{g h}(z)\right): \\
= & \sum_{n=-\infty}^{\infty} c_{-n} L_{n}^{(m)}+c_{0}\left[\sum_{n \geq 1} n\left(c_{-n} b_{n}+b_{-n} c_{n}\right)-1\right]-2 b_{0} \sum_{n \geq 1} n c_{-n} c_{n} \\
& -\sum_{n \geq 1, m \geq 1}\left[m\left(b_{-n-m} c_{n} c_{m}-c_{-n} c_{-m} b_{n+m}\right)\right. \\
& \left.+(n+2 m)\left(b_{-n} c_{-m} c_{m+n}+c_{-m-n} c_{m} b_{n}\right)\right]
\end{aligned}
$$

Thanks to the presence of $Y$ we can relate $g_{o}$ to the mass of D-brane $M$ [17]:

$$
\frac{1}{g_{o}^{2}}=2 \pi^{2} M
$$

We can calculate $M$ by computing the cylinder amplitude and factorizing the contribution of gravitons, or computing the disk partition function [19, 21]. The mass $M_{J}$ of the brane represented by the boundary state $|J\rangle$ is

$$
M_{J} \propto S_{J}^{0}=\sqrt{\frac{2}{k+2}} \sin \frac{(2 J+1) \pi}{k+2} .
$$

The proportionality coefficient is determined from the information of $\mathcal{M}$. We do not have to know it for our purpose.

The string field $\Psi$ can be expanded by primary operators and their descendants. We do not have to consider all of them for seeking lump solutions on D-branes which depends only on the $\mathrm{SU}(2)$ directions. The components which we have to consider are those made by acting with the oscillators

$$
\left\{J_{-1}^{a}, J_{-2}^{a}, \cdots ; L_{-2}^{\prime}, L_{-3}^{\prime}, \cdots ; c_{0}, c_{-1} \cdots ;, b_{-1}, b_{-2}, \cdots\right\}
$$

on $|j, m\rangle$, where $|j, m\rangle=\Phi_{j}^{m}(0) c_{1}|0\rangle$ and $|0\rangle$ is the $\operatorname{SL}(2, \mathrm{R})$ invariant vacuum. It can be easily shown that the set of the other components do not have linear terms in the action and therefore their equations of motion are satisfied if they vanish. Hence they can be taken to be zero from beginning. Then $\Psi$ can be expanded as follows.

$$
\begin{aligned}
\Psi= & |j, m\rangle t_{m}^{j} \\
& +J_{-1}^{a}|j, m\rangle u_{m}^{a j} \\
& +J_{-2}^{a}|j, m\rangle v_{m}^{a j}+J_{-1}^{a} J_{-1}^{b}|j, m\rangle v_{m}^{a b j}+b_{-1} c_{-1}|j, m\rangle \beta_{m}^{a j}+L_{-2}^{\prime}|j, m\rangle w_{m}^{j} \\
& +\cdots
\end{aligned}
$$




$$
\begin{aligned}
& +c_{0}\left[b_{-1}|j, m\rangle \widetilde{t}_{m}^{j}\right. \\
& +J_{-1}^{a} b_{-1}|j, m\rangle \widetilde{u}_{m}^{a j}+b_{-2}|j, m\rangle \widetilde{u}_{m}^{j} \\
& +\cdots]
\end{aligned}
$$

Here the repeated indices are summed over. The terms containing $c_{0}$ are removed if we take the Siegel gauge $b_{0} \Phi=0$. $|j, m\rangle$ and its descendants correspond to the spherical harmonic $Y_{j}^{m}$ [19, 25. Since $\left(Y_{j}^{m}\right)^{*}=(-1)^{m} Y_{j}^{-m}$, we impose the following reality conditions.

$$
\begin{aligned}
& \left(t_{m}^{j}\right)^{*}=(-1)^{m} t_{-m}^{j}, \quad\left(u_{m}^{a j}\right)^{*}=(-1)^{m} u_{-m}^{a j}, \quad\left(v_{m}^{a b j}\right)^{*}=(-1)^{m} v_{-m}^{a b j}, \\
& \left(\beta_{m}^{j}\right)^{*}=(-1)^{m} \beta_{-m}^{j}, \quad\left(w_{m}^{j}\right)^{*}=(-1)^{m} w_{-m}^{j}, \quad\left(\widetilde{u}_{m}^{a j}\right)^{*}=(-1)^{m} \widetilde{u}_{-m}^{a j}, \\
& \left(v_{m}^{a j}\right)^{*}=-(-1)^{m} v_{-m}^{a j}, \quad\left(\widetilde{t}_{m}^{j}\right)^{*}=-(-1)^{m} \widetilde{t}_{-m}^{j}, \quad\left(\widetilde{u}_{m}^{j}\right)^{*}=-(-1)^{m} \widetilde{u}_{-m}^{j}, \quad \text { etc. }
\end{aligned}
$$

Additional sign factor is needed for $v_{m}^{a j}, \widetilde{t}_{m}^{j}$ and $\widetilde{u}_{m}^{j}$ because their BPZ conjugations are equal to minus the hermitian conjugations.

We can reduce the number of components further. The components with the eigenvalues of $J_{0}^{3} \neq 0$ has no linear terms because of global SU(2) symmetry generated by $J_{0}^{a}$. Therefore we can satisfy the equations of motion by putting them to be zero. We consider only the components with $J_{0}^{3}=0$. Then we can restrict the components in (20) in the following form.

$$
\begin{aligned}
& t_{m}^{j}=t^{j} \delta_{0 m}, \quad \beta_{m}^{j}=\beta^{j} \delta_{0 m}, \quad w_{m}^{j}=w^{j} \delta_{0 m}, \quad \widetilde{t}_{m}^{j}=i \widetilde{t}^{j} \delta_{0 m}, \quad \widetilde{u}_{m}^{j}=i \widetilde{u}^{j} \delta_{0 m}, \\
& u_{m}^{a j}=\eta^{j+j^{\prime}+1} U^{m^{\prime} a}\left\langle 1, m^{\prime}, j, m \mid 1 j j^{\prime} 0\right\rangle u^{j j^{\prime}} \\
& v_{m}^{a j}=\eta^{j+j^{\prime}} U^{m^{\prime} a}\left\langle 1, m^{\prime}, j, m \mid 1 j j^{\prime} 0\right\rangle v^{j j^{\prime}} \\
& \widetilde{u}_{m}^{a j}=\eta^{j+j^{\prime}+1} U^{m^{\prime} a}\left\langle 1, m^{\prime}, j, m \mid 1 j j^{\prime} 0\right\rangle \widetilde{u}^{j j^{\prime}} \\
& v_{m}^{a b j}=\eta^{j+j^{\prime \prime}} U^{m_{3} b}\left\langle 1, m_{3}, j^{\prime}, m_{2} \mid 1 j^{\prime} j^{\prime \prime} 0\right\rangle U^{m_{1} a}\left\langle 1, m_{1}, j, m \mid 1 j j^{\prime} m_{2}\right\rangle v^{j j^{\prime} j^{\prime \prime}}
\end{aligned}
$$

where $\eta^{j}=1$ (for $j=$ even), $i$ (for $j=$ odd) and $U^{m a}$ is the following matrix which convert the standard spin 1 index $m$ to the adjoint index $a$.

$$
U^{m a}=\left(\begin{array}{ccc}
-\frac{1}{\sqrt{2}} & -\frac{i}{\sqrt{2}} & 0 \\
0 & 0 & 1 \\
\frac{1}{\sqrt{2}} & -\frac{i}{\sqrt{2}} & 0
\end{array}\right) .
$$

The reality condition for these components are determined from (21). All of them are real. i.e.

$$
\begin{aligned}
& \left(t^{j}\right)^{*}=t^{j}, \quad\left(\beta^{j}\right)^{*}=\beta^{j}, \quad\left(w^{j}\right)^{*}=w^{j}, \quad\left(\widetilde{t}^{j}\right)^{*}=\widetilde{t}^{j}, \quad\left(\widetilde{u}^{j}\right)^{*}=\widetilde{u}^{j}, \\
& \left(u^{j j^{\prime}}\right)^{*}=u^{j j^{\prime}}, \quad\left(v^{j j^{\prime}}\right)^{*}=v^{j j^{\prime}}, \quad\left(\widetilde{u}^{j j^{\prime}}\right)^{*}=\widetilde{u}^{j j^{\prime}}, \quad\left(v^{j j^{\prime} j^{\prime \prime}}\right)^{*}=v^{j j^{\prime} j^{\prime \prime}} .
\end{aligned}
$$


Now let us explain the calculation of the quadratic terms in the action. First, we get 2-point functions of two $\mathrm{SU}(2)$ primary operators by putting $j_{2}=m_{2}=0$ in eq. (10) $\left(\Phi_{0}^{0}=1\right)$.

$$
\begin{aligned}
\left\langle\Phi_{j}^{m} \mid \Phi_{j^{\prime}}^{m^{\prime}}\right\rangle & =C\left[\begin{array}{ccc}
m & 0 & m^{\prime} \\
j & 0 & j^{\prime}
\end{array}\right] \\
& =\frac{(-1)^{m}}{\sqrt{(2 j+1)[2 j+1]}} \delta_{j, j^{\prime}} \delta_{m,-m^{\prime}} .
\end{aligned}
$$

The BPZ conjugation of $J_{-n_{1}}^{a_{1}} J_{-n_{2}}^{a_{2}} \cdots\left|\Phi_{j}^{m}\right\rangle$ is $\left\langle\Phi_{j}^{m}\right| \cdots\left[(-1)^{n_{2}+1} J_{n_{2}}^{a_{2}}\right]\left[(-1)^{n_{1}+1} J_{n_{1}}^{a_{1}}\right]$. Using this and the commutation relation (3), 2-point functions of descendants can be reduced to those of primaries.

Let $\Psi_{1}=\phi_{1} G_{1}|0\rangle$ and $\Psi_{2}=\phi_{2} G_{2}|0\rangle$, where $G_{i}$ are ghost parts, and $\phi_{i}$ are SU(2) parts. Then

$$
\left\langle\Psi_{1}, Q_{B} \Psi_{2}\right\rangle=\oint \frac{d z}{2 \pi i}\left[\left\langle G_{1}|c(z)| G_{2}\right\rangle\left\langle\phi_{1}\left|T^{(m)}(z)\right| \phi_{2}\right\rangle+\frac{1}{2}\left\langle G_{1}\left|: c(z) T^{(g h)}(z):\right| G_{2}\right\rangle\left\langle\phi_{1} \mid \phi_{2}\right\rangle\right] .
$$

Since $\oint \frac{d z}{2 \pi i}: c(z) T^{(g h)}(z)$ : does not change descendant level and $\left\langle\phi_{1} \mid \phi_{2}\right\rangle$ is nonzero only when $\phi_{1}$ and $\phi_{2}$ have the same descendant level, the second term of (26) is nonzero only when $\Psi_{1}$ and $\Psi_{2}$ have the same $\mathrm{SU}(2)$ and ghost descendant level. $\left\langle\phi_{1}\left|T^{(m)}(z)\right| \phi_{2}\right\rangle$ is nonzero only when $T^{(m)}(z)\left|\phi_{2}\right\rangle$ and $\left|\phi_{1}\right\rangle$ have the same descendant level. Therefore $\left\langle\phi_{1}\left|T^{(m)}(z)\right| \phi_{2}\right\rangle=$ $z^{N_{1}-N_{2}-2}\left\langle\phi_{1}\left|L_{N_{2}-N_{1}}^{(m)}\right| \phi_{2}\right\rangle$, where $N_{i}$ are the descendant level of $\Psi_{i}$. Then the first term of (26) is equal to

$$
\left\langle G_{1}\left|c_{N_{1}-N_{2}}\right| G_{2}\right\rangle\left\langle\phi_{1}\left|L_{N_{2}-N_{1}}^{(m)}\right| \phi_{2}\right\rangle .
$$

This is nonzero only when $\Psi_{1}$ and $\Psi_{2}$ have the same total descendant level. Thus we can reduce the number of terms needed to calculate. Remaining terms can be calculated by using commutation relations of $L_{n}^{(m)}, J_{n}^{a}, c_{n}$ and $b_{n}$.

(26) becomes particularly simple when $\left|G_{1}\right\rangle$ and $\left|G_{2}\right\rangle$ do not contain $c_{0}$ i.e. $\Psi_{1}$ and $\Psi_{2}$ satisfy the Siegel gauge condition:

$$
\left\langle\Psi_{1}, Q_{B} \Psi_{2}\right\rangle=h\left\langle\phi_{1} \mid \phi_{2}\right\rangle\left\langle G_{1} \mid G_{2}\right\rangle,
$$

where $h$ is the dimension of $\Psi_{1}$.

Next we explain how to calculate the cubic terms in the action. We adopt the method given in [28. Let $\mathcal{K}$ be an operator and $v(z)$ a function which transforms as $f \circ \mathcal{K}(z)=$ $\left(f^{\prime}(z)\right)^{h} \mathcal{K}(f(z))+g(f(z), z)$ and $f \circ v(z)=\left(f^{\prime}(z)\right)^{1-h} v(f(z))$ under the conformal transformation $f$. One can think of $\mathcal{K}$ as $T, j$ (ghost number current), $b, c, J^{a}$ (WZW current) and so on. 
$g(f(z), z)$ represents anomaly in the case of $\mathcal{K}=T$ or $j$. We take $g(f(z), z)$ to be zero if $\mathcal{K}$ is Grassmann odd, and assume $v(z)$ to be regular at infinity. Then the conservation law for $\mathcal{K}$ is (for the notation see [28])

$$
\begin{aligned}
0= & \left\langle\left(\oint_{\mathcal{C}} \frac{d z}{2 \pi i} v(z) \mathcal{K}(z)-\oint_{\widetilde{z}=0} \frac{d \widetilde{z}}{2 \pi i} g(I(\widetilde{z}), \widetilde{z}) I \circ v(\widetilde{z})\right) f_{1} \circ \Phi_{1}(0) f_{2} \circ \Phi_{2}(0) f_{3} \circ \Phi_{3}(0)\right\rangle \\
= & \left\langle\left[\sum_{i=1}^{3}\left(\oint_{\mathcal{C}_{i}} \frac{d z_{i}}{2 \pi i} f_{i} \circ v\left(z_{i}\right) f_{i} \circ \mathcal{K}\left(z_{i}\right)-g\left(f_{i}\left(z_{i}\right), z_{i}\right) f_{i} \circ v\left(z_{i}\right)\right)-\oint_{\widetilde{z}=0} \frac{d \widetilde{z}}{2 \pi i} g(I(\widetilde{z}), \widetilde{z}) I \circ v(\widetilde{z})\right]\right. \\
& \left.\times f_{1} \circ \Phi_{1}(0) f_{2} \circ \Phi_{2}(0) f_{3} \circ \Phi_{3}(0)\right\rangle .
\end{aligned}
$$

The representation of this law by oscillators is

$$
\left\langle\left(\sum_{i=1}^{3} \sum_{n} \mathcal{K}_{n}^{(i)} v_{-n}^{(i)}-A(v)\right) \Phi_{1}, \Phi_{2}, \Phi_{3}\right\rangle=0,
$$

where

$$
\begin{aligned}
f_{i} \circ \mathcal{K}\left(z_{i}\right) & =\sum_{n} \mathcal{K}_{n}^{(i)} z_{i}^{-n-h}, \\
f_{i} \circ v\left(z_{i}\right) & =\sum_{n} v_{n}^{(i)} z_{i}^{-n+h-1},
\end{aligned}
$$

and

$$
\begin{aligned}
A(f, v) & =\oint_{z=0} \frac{d z}{2 \pi i} g(f(z), z) f \circ v(z) \\
A(v) & =\sum_{i=1}^{3} A\left(f_{i}, v\right)+A(I, v)
\end{aligned}
$$

We take $v(z)=z^{-l}\left(z^{2}-3\right)^{k}$ 28]. By using $f_{2}\left(z_{2}\right)=-f_{2}\left(-z_{2}\right)$ and $f_{3}\left(z_{3}\right)=-\left.f_{1}\left(-z_{1}\right)\right|_{z_{1}=z_{3}}$, we can derive $v_{l+h+2 n}^{(2)}=0, \quad v_{-k+h+n}^{(3)}=(-1)^{k+l+n+1} v_{-k+h+n}^{(1)} \quad(n \in \mathbf{Z})$, and from the explicit form of $v(z)$ the Laurent expansions of $f_{1} \circ v\left(z_{1}\right), f_{2} \circ v\left(z_{2}\right)$ and $f_{3} \circ v\left(z_{3}\right)$ start from $z_{1}^{k}, z_{2}^{-l}$ and $z_{3}^{k}$ respectively. We suppose $A(v)=0$ if $l+h=$ even. This is satisfied in the case of $\mathcal{K}=T$ or $j$. Then we can use the relation

$$
\begin{aligned}
\mathcal{K}_{-l-h+1}^{(2)}= & -\left(v_{l+h-1}^{(2)}\right)^{-1}\left[-A(v) \delta_{l+h, o d d}+\sum_{m=0}^{\infty} v_{l+h-3-2 m}^{(2)} \mathcal{K}_{-l-h+3+2 m}^{(2)}\right. \\
& \left.+\sum_{m=0}^{\infty} v_{-k+h-1-m}^{(1)}\left(\mathcal{K}_{k-h+1+m}^{(1)}+(-1)^{k+l-m} \mathcal{K}_{k-h+1+m}^{(3)}\right)\right]
\end{aligned}
$$

in the 3-point function, where $\delta_{l+h, \text { odd }}=1$ (for $l+h$ odd), $=0$ (for $l+h$ even or noninteger $)$. If we take $l \geq(>) 1-h$ and $k>(\geq) h-1$, mode numbers of $\mathcal{K}$ which appear in the right hand 
side of (35) are greater than that of the left hand side. Hence by using (35) iteratively, 3-point functions of descendants can be reduced to those of primaries, which are given in the $\mathrm{SU}(2)$ case as follows.

$$
\begin{aligned}
\left\langle\Phi_{j_{1}}^{m_{1}}, \Phi_{j_{2}}^{m_{2}}, \Phi_{j_{3}}^{m_{3}}\right\rangle & =\left(\frac{8}{3}\right)^{h_{1}+h_{3}}\left(\frac{2}{3}\right)^{h_{2}}\left\langle\Phi_{j_{1}}^{m_{1}}(\sqrt{3}) \Phi_{j_{2}}^{m_{2}}(0) \Phi_{j_{3}}^{m_{3}}(-\sqrt{3})\right\rangle \\
& =K^{-h_{j_{1}}-h_{j_{2}}-h_{j_{3}} C}\left[\begin{array}{ccc}
m_{1} & m_{2} & m_{3} \\
j_{1} & j_{2} & j_{3}
\end{array}\right]
\end{aligned}
$$

where $K=\frac{3 \sqrt{3}}{4}$.

$\left\langle\Psi_{1}, \Psi_{2}, \Psi_{3}\right\rangle$ has the cyclic symmetry: $\left\langle\Psi_{1}, \Psi_{2}, \Psi_{3}\right\rangle=(-1)^{\gamma^{3}\left(\gamma^{1}+\gamma^{2}\right)}\left\langle\Psi_{3}, \Psi_{1}, \Psi_{2}\right\rangle$, where $(-1)^{\gamma^{i}}$ is the Grassmann parity of $\Psi_{i}$. In addition to this, there is the following relation: if $\phi_{i}$ have Grassmann parity $(-1)^{g_{i}}$ and are level $N_{i}$ descendants of the primary operators $\Phi_{i}$ with Grasmann parity $(-1)^{G_{i}}$, and satisfy the relation $\left\langle\Phi_{1}, \Phi_{2}, \Phi_{3}\right\rangle=(-1)^{N_{123}+G_{1} G_{2}+G_{2} G_{3}+G_{3} G_{1}}\left\langle\Phi_{3}, \Phi_{2}, \Phi_{1}\right\rangle$, then

$$
\left\langle\phi_{1}, \phi_{2}, \phi_{3}\right\rangle=(-1)^{N_{123}+N_{1}+N_{2}+N_{3}+g_{1} g_{2}+g_{2} g_{3}+g_{3} g_{1}}\left\langle\phi_{3}, \phi_{2}, \phi_{1}\right\rangle .
$$

We can regard this relation as the result of worldsheet parity symmetry, and this can also be proven directly as follows by applying the above procedure. We use the induction on the descendant level of $\phi_{1,2,3}$ :

$$
\begin{aligned}
-v_{n}^{(2)}\left\langle\phi_{1}, \mathcal{K}_{-n} \phi_{2}, \phi_{3}\right\rangle= & -A(v) \delta_{n, \text { even }}\left\langle\phi_{1}, \phi_{2}, \phi_{3}\right\rangle \\
& +\sum_{m=0}^{\infty}\left[v_{n-2 m-2}^{(2)}\left\langle\phi_{1}, \mathcal{K}_{-n+2 m+2} \phi_{2}, \phi_{3}\right\rangle\right. \\
& +(-1)^{g_{\mathcal{K}} g_{1}} v_{-k+h-1-m}^{(1)}\left\langle\mathcal{K}_{k-h+1+m} \phi_{1}, \phi_{2}, \phi_{3}\right\rangle \\
& \left.+(-1)^{k-h+1+n+m+g_{\mathcal{K}} g_{2}} v_{-k+h-1-m}^{(1)}\left\langle\phi_{1}, \phi_{2}, \mathcal{K}_{k-h+1+m} \phi_{3}\right\rangle\right] \\
= & -(-1)^{N_{123}+N_{1}+N_{2}+N_{3}+g_{1} g_{2}+g_{2} g_{3}+g_{3} g_{1}} A(v) \delta_{n, \text { even }}\left\langle\phi_{3}, \phi_{2}, \phi_{1}\right\rangle \\
& +\sum_{m=0}^{\infty}\left[(-1)^{N_{123}+N_{1}+N_{2}+N_{3}+g_{1}\left(g_{2}+g_{\mathcal{K}}\right)+\left(g_{2}+g_{\mathcal{K}}\right) g_{3}+g_{3} g_{1}+n-2 m-2}\right. \\
& \times v_{n-2 m-2}^{(2)}\left\langle\phi_{3}, \mathcal{K}_{-n+2 m+2} \phi_{2}, \phi_{1}\right\rangle \\
& +(-1)^{N_{123}+N_{1}+N_{2}+N_{3}+\left(g_{1}+g_{\mathcal{K}}\right) g_{2}+g_{2} g_{3}+g_{3}\left(g_{1}+g_{\mathcal{K}}\right)+g_{\mathcal{K}} g_{1}-k+h-1-m} \\
& \times v_{-k+h-1-m}^{(1)}\left\langle\phi_{3}, \phi_{2}, \mathcal{K}_{k-h+1+m} \phi_{1}\right\rangle \\
& +(-1)^{N_{123}+N_{1}+N_{2}+N_{3}+g_{1} g_{2}+g_{2}\left(g_{3}+g_{\mathcal{K}}\right)+\left(g_{3}+g_{\mathcal{K}}\right) g_{1}+n+g_{\mathcal{K}} g_{2}} \\
& \left.\times v_{-k+h-1-m}^{(1)}\left\langle\mathcal{K}_{k-h+1+m} \phi_{3}, \phi_{2}, \phi_{1}\right\rangle\right] \\
= & (-1)^{N_{123}+N_{1}+\left(N_{2}+n\right)+N_{3}+g_{1}\left(g_{2}+g_{\mathcal{K}}\right)+\left(g_{2}+g_{\mathcal{K}}\right) g_{3}+g_{3} g_{1}}\left[-A(v) \delta_{n, e v e n}\left\langle\phi_{3}, \phi_{2}, \phi_{1}\right\rangle\right.
\end{aligned}
$$




$$
\begin{aligned}
& +\sum_{m=0}^{\infty}\left[v_{n-2 m-2}^{(2)}\left\langle\phi_{3}, \mathcal{K}_{-n+2 m+2} \phi_{2}, \phi_{1}\right\rangle\right. \\
& +(-1)^{g_{\mathcal{K}} g_{3}} v_{-k+h-1-m}^{(1)}\left\langle\mathcal{K}_{k-h+1+m} \phi_{3}, \phi_{2}, \phi_{1}\right\rangle \\
& \left.\left.+(-1)^{g_{\mathcal{K}} g_{2}-k+h-1-n-m} v_{-k+h-1-m}^{(1)}\left\langle\phi_{3}, \phi_{2}, \mathcal{K}_{k-h+1+m} \phi_{1}\right\rangle\right]\right] \\
& =-v_{n}^{(2)}(-1)^{N_{123}+N_{1}+\left(N_{2}+n\right)+N_{3}+g_{1}\left(g_{2}+g_{\mathcal{K}}\right)+\left(g_{2}+g_{\mathcal{K}}\right) g_{3}+g_{3} g_{1}}\left\langle\phi_{3}, \mathcal{K}_{-n} \phi_{2}, \phi_{1}\right\rangle .
\end{aligned}
$$

Here we used the assumption of the induction and $A(v)=0$ when $g_{\mathcal{K}}($ Grasmann parity of $\mathcal{K})=$ 1. In addition, by using the cyclic symmetry,

$$
\begin{aligned}
& \left\langle\phi_{1}, \phi_{2}, \mathcal{K}_{-n} \phi_{3}\right\rangle=(-1)^{N_{123}+N_{1}+N_{2}+\left(N_{3}+n\right)+g_{1} g_{2}+g_{2}\left(g_{3}+g_{\mathcal{K}}\right)+\left(g_{3}+g_{\mathcal{K}}\right) g_{1}}\left\langle\mathcal{K}_{-n} \phi_{3}, \phi_{2}, \phi_{1}\right\rangle, \\
& \left\langle\mathcal{K}_{-n} \phi_{1}, \phi_{2}, \phi_{3}\right\rangle=(-1)^{N_{123}+\left(N_{1}+n\right)+N_{2}+N_{3}+\left(g_{1}+g_{\mathcal{K}}\right) g_{2}+g_{2} g_{3}+g_{3}\left(g_{1}+g_{\mathcal{K}}\right)}\left\langle\phi_{3}, \phi_{2}, \mathcal{K}_{-n} \phi_{1}\right\rangle .
\end{aligned}
$$

Hence we conclude that (37) is valid by induction. We can use (37) and the cyclic symmetry to reduce the number of terms in the action necessary for our calculation. In the $\mathrm{SU}(2)$ case, $N_{123}=j_{1}+j_{2}+j_{3}$ for $\Phi_{i}=\Phi_{j_{i}}^{m_{i}}$, as can be seen from (13).

As an example, let us calculate $\left\langle J_{-1}^{a} \Phi_{j_{1}}^{m_{1}}, J_{-1}^{b} \Phi_{j_{2}}^{m_{2}}, \Phi_{j_{3}}^{m_{3}}\right\rangle$ by using the eq.(4.18) of [28] for WZW current $J^{a}: J_{-1}^{(2) a}=-\left(\frac{5}{27} J_{1}^{(2) a}+\cdots\right)+\left(-\frac{2}{3 \sqrt{3}} J_{0}^{(1) a}+\frac{16}{27} J_{1}^{(1) a}+\cdots\right)+\left(\frac{2}{3 \sqrt{3}} J_{0}^{(3) a}+\frac{16}{27} J_{1}^{(3) a}+\cdots\right)$.

$$
\begin{aligned}
& \left\langle J_{-1}^{a} \Phi_{j_{1}}^{m_{1}}, J_{-1}^{b} \Phi_{j_{2}}^{m_{2}}, \Phi_{j_{3}}^{m_{3}}\right\rangle \\
= & -\frac{2}{3 \sqrt{3}}\left\langle J_{0}^{b} J_{-1}^{a} \Phi_{j_{1}}^{m_{1}}, \Phi_{j_{2}}^{m_{2}}, \Phi_{j_{3}}^{m_{3}}\right\rangle \\
& +\frac{16}{27}\left\langle J_{1}^{b} J_{-1}^{a} \Phi_{j_{1}}^{m_{1}}, \Phi_{j_{2}}^{m_{2}}, \Phi_{j_{3}}^{m_{3}}\right\rangle \\
& +\frac{2}{3 \sqrt{3}}\left\langle J_{-1}^{a} \Phi_{j_{1}}^{m_{1}}, \Phi_{j_{2}}^{m_{2}}, J_{0}^{b} \Phi_{j_{3}}^{m_{3}}\right\rangle \\
= & -\frac{2}{3 \sqrt{3}} i f^{b a c}\left\langle J_{-1}^{c} \Phi_{j_{1}}^{m_{1}}, \Phi_{j_{2}}^{m_{2}}, \Phi_{j_{3}}^{m_{3}}\right\rangle-\frac{2}{3 \sqrt{3}}\left\langle J_{-1}^{a} \Phi_{j_{1}}^{m_{1}^{\prime}}, \Phi_{j_{2}}^{m_{2}}, \Phi_{j_{3}}^{m_{3}}\right\rangle\left(T_{\left(j_{1}\right)}^{b}\right)_{m_{1}^{\prime}}^{m_{1}} \\
& +\frac{16}{27} i f^{b a c}\left\langle J_{0}^{c} \Phi_{j_{1}}^{m_{1}}, \Phi_{j_{2}}^{m_{2}}, \Phi_{j_{3}}^{m_{3}}\right\rangle+\frac{16}{27} k \delta^{a b}\left\langle\Phi_{j_{1}}^{m_{1}}, \Phi_{j_{2}}^{m_{2}}, \Phi_{j_{3}}^{m_{3}}\right\rangle \\
& +\frac{2}{3 \sqrt{3}}\left\langle J_{-1}^{a} \Phi_{j_{1}}^{m_{1}}, \Phi_{j_{2}}^{m_{2}}, \Phi_{j_{3}}^{m_{3}^{\prime}}\right\rangle\left(T_{\left(j_{3}\right)}^{b}\right)_{m_{3}^{\prime}}^{m_{3}} \\
= & \frac{2}{3 \sqrt{3}} i f^{b a c}\left(\frac{2}{3 \sqrt{3}}\right)\left\langle\Phi_{j_{1}}^{m_{1}}, J_{0}^{c} \Phi_{j_{2}}^{m_{2}}, \Phi_{j_{3}}^{m_{3}}\right\rangle-\frac{2}{3 \sqrt{3}} i f^{b a c}\left(-\frac{2}{3 \sqrt{3}}\right)\left\langle\Phi_{j_{1}}^{m_{1}}, \Phi_{j_{2}}^{m_{2}}, J_{0}^{c} \Phi_{j_{3}}^{m_{3}}\right\rangle \\
& -\frac{2}{3 \sqrt{3}}\left(\frac{2}{3 \sqrt{3}}\right)\left\langle\Phi_{j_{1}}^{m_{1}^{\prime}}, J_{0}^{a} \Phi_{j_{2}}^{m_{2}}, \Phi_{j_{3}}^{m_{3}}\right\rangle\left(T_{\left(j_{1}\right)}^{b}\right)_{m_{1}^{\prime}}^{m_{1}}-\frac{2}{3 \sqrt{3}}\left(-\frac{2}{3 \sqrt{3}}\right)\left\langle\Phi_{j_{1}}^{m_{1}^{\prime}}, \Phi_{j_{2}}^{m_{2}}, J_{0}^{a} \Phi_{j_{3}}^{m_{3}^{\prime}}\right\rangle\left(T_{\left(j_{1}\right)}^{b}\right)_{m_{1}^{\prime}}^{m_{1}} \\
& +\frac{16}{27} i f b a c\left\langle\Phi_{j_{1}}^{m_{1}^{\prime}}, \Phi_{j_{2}}^{m_{2}}, \Phi_{j_{3}}^{m_{3}}\right\rangle\left(T_{\left(j_{1}\right)}^{c}\right)_{m_{1}^{\prime}}^{m_{1}}+\frac{16}{27} k \delta^{a b}\left\langle\Phi_{j_{1}}^{m_{1}}, \Phi_{j_{2}}^{m_{2}}, \Phi_{j_{3}}^{m_{3}}\right\rangle \\
& +\frac{2}{3 \sqrt{3}}\left(\frac{2}{3 \sqrt{3}}\right)\left\langle\Phi_{j_{1}}^{m_{1}}, J_{0}^{a} \Phi_{j_{2}}^{m_{2}}, \Phi_{j_{3}}^{m_{3}^{\prime}}\right\rangle\left(T_{\left(j_{3}\right)}^{b}\right)_{m_{3}^{\prime}}^{m_{3}}+\frac{2}{3 \sqrt{3}}\left(-\frac{2}{3 \sqrt{3}}\right)\left\langle\Phi_{j_{1}}^{m_{1}}, \Phi_{j_{2}}^{m_{2}}, J_{0}^{a} \Phi_{j_{3}}^{m_{3}^{\prime}}\right\rangle\left(T_{\left(j_{3}\right)}^{b}\right)_{m_{3}^{\prime}}^{m_{3}}
\end{aligned}
$$




$$
=\frac{4}{27}\left\langle\Phi_{j_{1}}, \Phi_{j_{2}}, \Phi_{j_{3}}\right\rangle\left[T_{3}^{a} T_{1}^{b}-T_{2}^{a} T_{1}^{b}+T_{2}^{a} T_{3}^{b}-T_{3}^{a} T_{3}^{b}+i f^{a b c}\left(T_{2}^{c}-T_{3}^{c}-4 T_{1}^{c}\right)+4 k \delta^{a b}\right] .
$$

In the last line we suppressed the indices $m$ and denote $T_{\left(j_{i}\right)}^{a}$ by $T_{i}^{a}$. At first sight this result does not satisfy the relation (37). However we can rewrite it to an explicitly symmetric form by using the Ward identity $\left\langle\Phi_{j_{1}}, \Phi_{j_{2}}, \Phi_{j_{3}}\right\rangle\left(T_{1}^{a}+T_{2}^{a}+T_{3}^{a}\right)=0$ :

$$
\left\langle J_{-1}^{a} \Phi_{j_{1}}^{m_{1}}, J_{-1}^{b} \Phi_{j_{2}}^{m_{2}}, \Phi_{j_{3}}^{m_{3}}\right\rangle=-\frac{4}{27}\left\langle\Phi_{j_{1}}, \Phi_{j_{2}}, \Phi_{j_{3}}\right\rangle\left[2 T_{1}^{a} T_{1}^{b}+2 T_{2}^{b} T_{2}^{a}+4 T_{1}^{b} T_{2}^{a}+T_{2}^{b} T_{1}^{a}+2 i f^{a b c}\left(T_{1}^{c}-T_{2}^{c}\right)-4 k \delta^{a b}\right] .
$$

We collect the results of the calculation of cubic terms in appendix B and the terms in the action necessary for our calculation are in appendix $\mathrm{C}$.

\section{$3 \quad$ Lump Solutions}

In this section we construct lump solutions and compare their energies with the expected values. We denote by $[N, M]$ the truncation that we consider only fields with the level (i.e. conformal dimension +1$)<N$ and retain only the terms with the sum of the levels $<M$.

The D2-brane corresponding to the boundary state $|J\rangle$ can be regarded as the bound state of $(2 J+1)$ D0-branes [21, 19], and D0-branes are conjectured to be able to vanish into the vacuum by tachyon condensation. Therefore one can imagine the process that some of the D0-branes forming the D2-brane vanish and the other D0-branes remain. Hence it is natural to conjecture that there exist lump solutions on D2-branes which represent $n$ D0branes $(n<2 J+1)$. The energy $V(\mathcal{T})$ of a classical configuration $\mathcal{T}$ is minus the action:

$$
\begin{aligned}
V(\mathcal{T}) & =-S(\mathcal{T})=\frac{1}{g_{o}^{2}}\left(-g_{o}^{2} S(\mathcal{T})\right)=2 \pi^{2} M_{J}\left(-g_{o}^{2} S(\mathcal{T})\right) \\
& \equiv M_{J} f(\mathcal{T})
\end{aligned}
$$

For the solution $\mathcal{T}_{\text {vac }}$ corresponding to the vacuum, it is conjectured that $f\left(\mathcal{T}_{\text {vac }}\right)=-1$. It can be expected that there exist the solutions $\mathcal{T}_{n}$ representing $n$ D0-branes (in this sense $\mathcal{T}_{\text {vac }}=\mathcal{T}_{0}$ ) and the difference between $V\left(\mathcal{T}_{n}\right)$ and $V\left(\mathcal{T}_{\text {vac }}\right)$ is equal to $n M_{0}$ :

$$
\begin{aligned}
V\left(\mathcal{T}_{n}\right) & =V\left(\mathcal{T}_{\text {vac }}\right)+n M_{0} \\
& =M_{J}\left(f\left(\mathcal{T}_{\text {vac }}\right)+n \cdot \frac{M_{0}}{M_{J}}\right) \\
& =M_{J}\left(f\left(\mathcal{T}_{\text {vac }}\right)+n \cdot \frac{\sin \frac{\pi}{k+2}}{\sin \frac{(2 J+1) \pi}{k+2}}\right) .
\end{aligned}
$$


Hence,

$$
f\left(\mathcal{T}_{n}\right)=f\left(\mathcal{T}_{\text {vac }}\right)+n \cdot \frac{\sin \frac{\pi}{k+2}}{\sin \frac{(2 J+1) \pi}{k+2}}=-1+n \cdot \frac{\sin \frac{\pi}{k+2}}{\sin \frac{(2 J+1) \pi}{k+2}} .
$$

We denote $f$ and $\mathcal{T}_{n}$ calculated in level $[N, M]$ truncation by $f^{[N, M]}$ and $\mathcal{T}_{n}^{[N, M]}$, and define $R_{n}^{[N, M]}$ as follows.

$$
R_{n}^{[N, M]} \equiv \frac{f^{[N, M]}\left(\mathcal{T}_{n}^{[N, M]}\right)}{f\left(\mathcal{T}_{n}\right)}
$$

$R_{n}^{[N, M]}$ is a function of $k$. We will calculate $R_{n}^{[N, M]}$ at various values of $k$ and see to what extent it is close to 1 .

Note that the configuration given by $\mathrm{SU}(2)$ transformation (generated by $J_{0}^{a}$ ) of a solution is also a solution. For lump solutions, these degrees of freedom correspond to the positions of D0-branes.

We will consider the cases with small $J$. If $J=0$ (i.e. D0-brane) there are no nontrivial primary operators and it is impossible to construct lump solution.

Then we will consider the following two cases.

- $J=1 / 2, k \geq 2$

- $J=1, k \geq 4$

In the first case, primary operators on the D2-brane are $\Phi_{0}^{0}$ and $\Phi_{1}^{m}$. (If $k=1$ only $\Phi_{0}^{0}$ appears.) Their dimensions are $h_{0}=0, h_{1}=\frac{2}{k+2}$. This case corresponds to the bound state of 2 D0-branes and we expect the existence of the solution $\mathcal{T}_{1}$.

In the second case, primary operators are $\Phi_{0}^{0}, \Phi_{1}^{m}$ and $\Phi_{2}^{m}$. (If $k \leq 3$ some of them do not appear and this case reduces to the previous one.) Their dimensions are $h_{0}, h_{1}, h_{2}=\frac{6}{k+2}$. This case corresponds to the bound state of 3 D0-branes and we expect the existence of the solutions $\mathcal{T}_{1}$ and $\mathcal{T}_{2}$.

We calculate the action in level $[1,3],[2,6]$ and $[5 / 2,5]$. The actual calculation is done by symbolic manipulation program Mathematica. 


\section{1 level $[1,3], k \rightarrow \infty, J=$ fixed}

In this case $\Psi=|j, m\rangle t_{m}^{j}$. The action is

$$
\frac{f^{[1,3]}}{2 \pi^{2}}=\frac{1}{2} \frac{(-1)^{m}}{\sqrt{(2 j+1)[2 j+1]}}\left(h_{j}-1\right) t_{-m}^{j} t_{m}^{j}+\frac{1}{3} K^{3-h_{j_{1}}-h_{j_{2}}-h_{j_{3}}} C\left[\begin{array}{ccc}
m_{1} & m_{2} & m_{3} \\
j_{1} & j_{2} & j_{3}
\end{array}\right] t_{m_{1}}^{j_{1}} t_{m_{2}}^{j_{2}} t_{m_{3}}^{j_{3}} .
$$

First, let us consider the case where $k \rightarrow \infty$ and $J=$ fixed. In this case it has been shown 18 that the structure of fuzzy spheres appears as follows. The spectrum of primary operator is $\Phi_{j}^{m}, \quad j=0,1, \cdots 2 J$ and the number of degrees of freedom of $t_{m}^{j}$ is $1+3+\cdots+(2 \cdot 2 J+1)=$ $(2 J+1) \times(2 J+1)$. These form a $(2 J+1) \times(2 J+1)$ matrix $t$ :

$$
(t)_{m_{1} m_{2}}=\sum_{j, m} \sqrt{\frac{2 J+1}{2 j+1}}(-1)^{j} t_{m}^{j}\left(T_{j, m}\right)_{m_{1} m_{2}},
$$

and 29]

$$
\left(T_{j, m}\right)_{m_{1} m_{2}}=(-1)^{J-m_{2}} \sqrt{2 j+1}\left\langle J, m_{2}, J,-m_{1} \mid J, J, j,-m\right\rangle .
$$

Then the terms in the action are realized as follows.

$$
\begin{aligned}
& \frac{1}{2 J+1} \operatorname{tr}(t t)=\sum_{j_{1}, j_{2}, j_{3}, m_{1}, m_{2}, m_{3}} C\left[\begin{array}{ccc}
m_{1} & m_{2} & m_{3} \\
j_{1} & j_{2} & j_{3}
\end{array}\right]_{k \rightarrow \infty} t_{m_{1}}^{j_{1}} t_{m_{2}}^{j_{2}} t_{m_{3}}^{j_{3}}, \\
& \frac{1}{2 J+1} \operatorname{tr}(t t)=\sum_{j_{1}, j_{2}, m_{1}, m_{2}} C\left[\begin{array}{ccc}
m_{1} & 0 & m_{2} \\
j_{1} & 0 & j_{2}
\end{array}\right]_{k \rightarrow \infty} t_{m_{1}}^{j_{1}} t_{m_{2}}^{j_{2}} \text {. }
\end{aligned}
$$

Hence,

$$
\frac{f^{[1,3]}}{2 \pi^{2}}=\frac{1}{2 J+1} \operatorname{tr}\left(-\frac{1}{2} t t+\frac{1}{3} K^{3} t t t\right) .
$$

Note that the form appearing in the trace is the same as the tachyon potential in level $(0,0)$.

Solutions of the equation of motion $-t+K^{3} t^{2}=0$ for this action can be constructed by introducing projection operators as in [30]:

$$
t=\lambda P, \quad P^{2}=P, \quad \operatorname{tr} P=(2 J+1)-n,
$$

and $\lambda$ is the nontrivial solution of $-\lambda+K^{3} \lambda^{2}=0$ i.e. $\lambda=K^{-3} . P^{2}=P$ and $\operatorname{tr} P=2 J+1-n$ means that if we diagonalize $P$ then $2 J+1-n$ eigenvalues are 1 and $n$ eigenvalues are 0 and therefore we can interpret this solution as $\mathcal{T}_{n}^{[1,3]}$. The energy of this solution is

$$
\begin{aligned}
\frac{f^{[1,3]}\left(\mathcal{T}_{n}^{[1,3]}\right)}{2 \pi^{2}} & =\frac{1}{2 J+1}\left(-\frac{1}{2} \lambda^{2}+\frac{1}{3} K^{3} \lambda^{3}\right) \operatorname{tr} P \\
& =-\frac{1}{2 J+1} \cdot \frac{1}{6} K^{-6}(2 J+1-n) .
\end{aligned}
$$

In this case $f\left(\mathcal{T}_{n}\right)=-\frac{1}{2 J+1}(2 J+1-n)$, and $R_{n}^{[1,3]}=\frac{\pi^{2}}{3 K^{6}} \simeq 0.684616$.

In the following we look for the sequences of solutions $R_{n}^{[1,3]}$ of which at $k \rightarrow \infty$ is 0.684616 . 


\section{2 level $[1,3], J=1 / 2$}

In this case we can easily calculate the action without dropping $J_{0}^{3} \neq 0$ components:

$$
\frac{f^{[1,3]}}{2 \pi^{2}}=-\frac{1}{2}\left(t_{0}^{0}\right)^{2}-\frac{k}{k+2} \frac{1}{\sqrt{3[3]}}\left|t_{m}^{1}\right|^{2}+\frac{1}{3} K^{3}\left(t_{0}^{0}\right)^{3}+\frac{1}{\sqrt{3[3]}} K^{3-4 /(k+2)}\left|t_{m}^{1}\right|^{2} t_{0}^{0} .
$$

We can easily solve the equations of motion and we find two solutions. One solution corresponds to the closed string vacuum, in which the components corresponding to nontrivial primary operators and their descendants vanishes. The other solution is

$$
\begin{aligned}
t_{0}^{0} & =\frac{k}{2(k+2)} K^{4 /(k+2)-3}, \\
\left|t_{m}^{1}\right|^{2} & =\sqrt{3[3]} \frac{k}{2(k+2)} K^{8 /(k+2)-6}\left(1-\frac{k}{2(k+2)} K^{4 /(k+2)}\right),
\end{aligned}
$$

which is correspond to $\mathcal{T}_{1}^{[1,3]}$.

$$
\frac{f^{[1,3]}\left(\mathcal{T}_{1}^{[1,3]}\right)}{2 \pi^{2}}=\frac{k^{2}}{4(k+2)^{2}} K^{8 /(k+2)-6}\left(-\frac{1}{2}+\frac{k}{6(k+2)} K^{4 /(k+2)}\right) .
$$

$R_{1}^{[1,3]}$ is shown in fig. 1. The value for $k \rightarrow \infty$ is 0.684616 , which agrees with the value expected above. The minimum is 0.554151 at $k=3$.

By $\mathrm{SU}(2)$ rotation, we can put $t_{ \pm 1}^{1}=0$. Then $T(\theta) \equiv \sqrt{\frac{4 \pi}{(2 j+1)^{1 / 2}[2 j+1]^{1 / 2}}}(-1)^{j} t_{m}^{j} Y_{j}^{m}(\theta)$ is not dependent on $\phi$, and its profile is shown in fig.2. Here we choose $t_{0}^{1}$ positive. (The factor $\sqrt{\frac{4 \pi}{(2 j+1)^{1 / 2}[2 j+1]^{1 / 2}}}(-1)^{j}$ is present because $\Phi_{j}^{m}$ is corresponding to $\sqrt{\frac{4 \pi}{(2 j+1)^{1 / 2}[2 j+1]^{1 / 2}}}(-1)^{j} Y_{j}^{m}$, which is obtained by comparing $\int d \Omega(-1)^{m^{\prime}} Y_{j^{\prime}}^{-m^{\prime}} Y_{j}^{m}=\delta_{j j^{\prime}} \delta_{m m^{\prime}}$ with $\left\langle(-1)^{m^{\prime}} \Phi_{j^{\prime}}^{-m^{\prime}} \Phi_{j}^{m}\right\rangle=$ $\delta_{j j^{\prime}} \delta_{m m^{\prime}} \sqrt{\frac{(2 j+1)^{1 / 2}[2 j+1]^{1 / 2}}{4 \pi}}$, and referring to (48).) It seems that this solution is not well localized. But this is not a problem since the radius of $S^{2}$ on which the D2-brane is wrapped is $\sim$ $\sqrt{k} \sin \frac{2 \pi J}{k}$ and this has the maximum $2 \sqrt{J}$ at $k=8 J$. This is sufficiently small for small $J$. Therefore the worldvolume of the D2-brane itself is very small. At any rate we cannot construct well localized configuration by using only $Y_{0}^{0}=\frac{1}{4 \pi}, Y_{1}^{0}=\frac{3}{4 \pi} \cos \theta$ and $Y_{1}^{ \pm 1}=\mp \frac{3}{8 \pi} \sin \theta e^{ \pm i \theta}$.

If we choose the sign of $t_{0}^{1}$ negative, the profile is given by $T(\pi-\theta)$. These two configurations seem to represent D0-branes at $\theta=0$ and $\pi$.

\section{3 level $[1,3], J=1$}

In this case we put $J_{0}^{3}=0$ components zero. Then

$$
\frac{f^{[1,3]}}{2 \pi^{2}}=-\frac{1}{2}\left(t^{0}\right)^{2}+\frac{1}{2} \frac{1}{\sqrt{3[3]}}\left(-1+\frac{2}{2+k}\right)\left(t^{1}\right)^{2}+\frac{1}{2} \frac{1}{\sqrt{5[5]}}\left(-1+\frac{6}{2+k}\right)\left(t^{2}\right)^{2}
$$




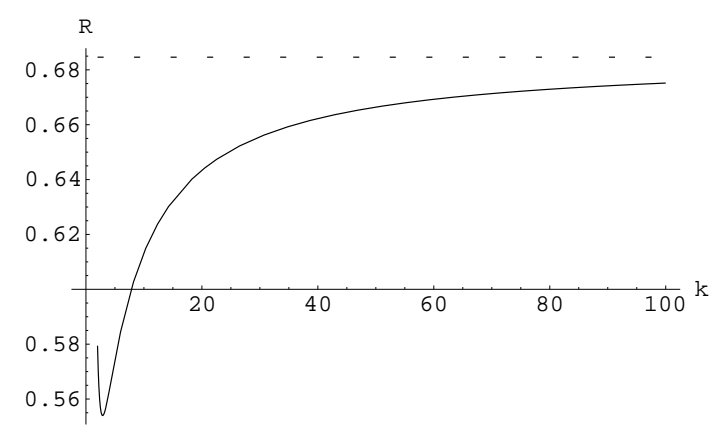

Figure 1: $R_{1}^{[1,3]}\left(J=\frac{1}{2}, k \geq 2\right)$

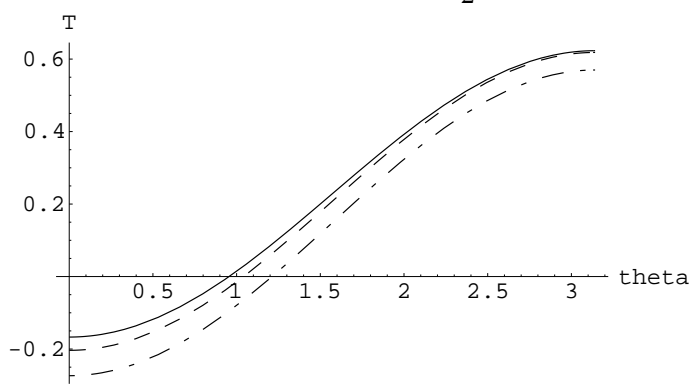

Figure 2: $T(\theta)$ (level [1,3], $\left.J=\frac{1}{2}\right) \quad k=100000000$ (solid line), $k=10$ (dashed line), $k=2$ (dot-dashed line) 


$$
\begin{aligned}
& +\frac{1}{3} K^{3}\left(t^{0}\right)^{3}+\frac{1}{\sqrt{3[3]}} K^{3-\frac{4}{k+2}}\left(t^{0}\right)\left(t^{1}\right)^{2}+\frac{1}{\sqrt{5[5]}} K^{3-\frac{12}{2+k}}\left(t^{0}\right)\left(t^{2}\right)^{2} \\
& +\sqrt{\frac{2}{3}} \frac{1}{\sqrt{5[5]}} c_{11}^{2} K^{3-\frac{10}{k+2}}\left(t^{1}\right)^{2}\left(t^{2}\right)-\frac{1}{3} \sqrt{\frac{2}{7}} \frac{1}{\sqrt{5[5]}} c_{22}^{2} K^{3-\frac{18}{k+2}}\left(t^{2}\right)^{3} .
\end{aligned}
$$

If $k=4, t^{2}$ must be put zero since the dimension of $\Phi_{2}^{m}$ is 1 . In the case with $k \neq 4$, we can reduce the equations of motion to quadratic equations, and for $k \geq 6$ we can find essentially two solutions which have $R_{1}^{[1,3]} \simeq 0.684616$ and $R_{2}^{[1,3]} \simeq 0.684616$ at $\infty$ respectively. The case with $k=4$ and 5 is somewhat special. we find only one nontrivial solution, which seems to be correspond to $\mathcal{T}_{1}^{[1,3]}\left(R_{1}^{[1,3]}=0.475766\right.$ for $k=4$ and $R_{1}^{[1,3]}=0.641119$ for $\left.k=5\right)$. We do not find any solution corresponding to $\mathcal{T}_{2}^{[1,3]}$.

$R_{1,2}^{[1,3]}$ and the profiles of $T(\theta) \equiv \sqrt{\frac{4 \pi}{(2 j+1)^{1 / 2}[2 j+1]^{1 / 2}}}(-1)^{j} t_{m}^{j} Y_{j}^{m}(\theta)$ are shown in fig. 3, 4, 5, 6. The minimum of $R_{1}^{[1,3]}$ and $R_{2}^{[1,3]}$ is 0.623983 at $k=7$ and 0.409721 at $k=6$ respectively.

\section{4 level $[2,6]$}

In this case, we can put $\Psi=|j, m\rangle t_{m}^{j}+J_{-1}^{a}|j, m\rangle u_{m}^{a j}+c_{0} b_{-1}|j, m\rangle \widetilde{t}_{m}^{j}$. The action is too long and it is not illuminating to write it here. Therefore we describe only its salient features.

$\widetilde{t}_{m}^{j}$ can be taken to be zero in the Siegel gauge. However their equations of motion may give nontrivial constraints [14. The linear term in $\widetilde{t}_{m}^{j}$ in the action is

$$
\frac{(-1)^{m}}{\sqrt{(2 j+1)[2 j+1]}} \widetilde{t}_{-m}^{j}\left(T_{j}^{a}\right)_{m}^{m^{\prime}} u_{m^{\prime}}^{a j}
$$

This term vanishes in our case since $\left(T_{j}^{a}\right)_{0} m^{\prime} u_{m^{\prime}}^{a j} \sim u^{j j}=0$ as can be seen from the results below. Therefore we can put $\widetilde{t}_{0}^{j}=0$ to satisfy their equations of motion and can adopt Siegel gauge condition for looking for lump solutions.

In this case we cannot expect any improvement at $k \rightarrow \infty$ comparing with the results of level $[1,3]$. The reason is as follows. The linear term in $u_{m}^{a j}$ is

$$
\frac{2}{3 \sqrt{3}} K^{3-h_{j_{1}}-h_{j_{2}}-h_{j_{3}}} C\left[\begin{array}{ccc}
m_{1} & m_{2} & m_{3} \\
j_{1} & j_{2} & j_{3}
\end{array}\right]\left(\left(T_{j_{3}}^{a}\right)_{m_{3}}^{m_{3}^{\prime}} \delta_{m_{1}}^{m_{1}^{\prime}}-\left(T_{j_{1}}^{a}\right)_{m_{1}}^{m_{1}^{\prime}} \delta_{m_{3}}^{m_{3}^{\prime}}\right) t_{m_{1}^{\prime}}^{j_{1}} u_{m_{2}}^{a j_{2}} t_{m_{3}^{\prime}}^{j_{3}}
$$

and at $k \rightarrow \infty$ the most dominant quadratic term in $u_{m}^{a j}$ is given from the last term of eq. (42):

$$
\frac{16}{27} k \cdot K^{3-h_{j_{1}}-h_{j_{2}}-h_{j_{3}} C}\left[\begin{array}{ccc}
m_{1} & m_{2} & m_{3} \\
j_{1} & j_{2} & j_{3}
\end{array}\right] u_{m_{1}}^{a j_{1}} u_{m_{2}}^{a j_{2}} t_{m_{3}}^{j_{3}}
$$




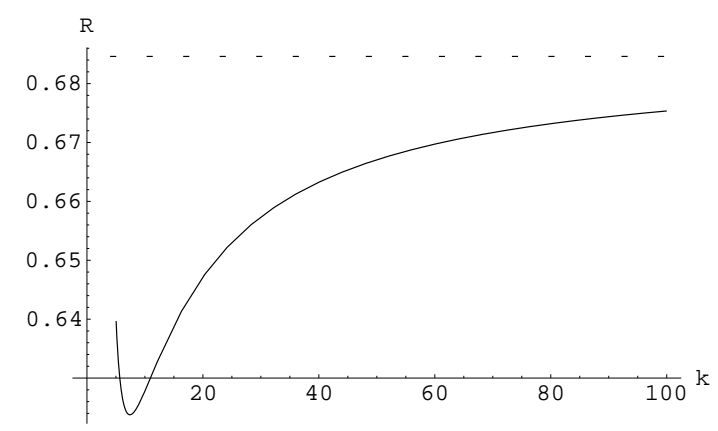

Figure $3: R_{1}^{[1,3]}(J=1, k \geq 4)$
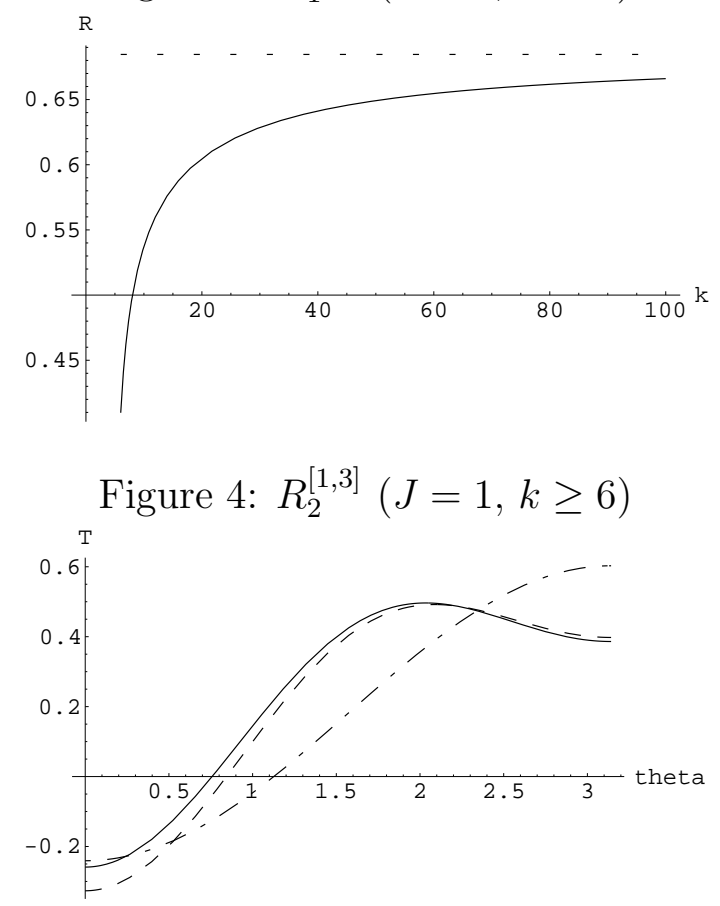

Figure 5: $T(\theta)$ (level $\left.[1,3], J=1, \mathcal{T}_{1}\right) \quad k=100000000$ (solid line), $k=10$ (dashed line), $k=4$ (dot-dashed line)

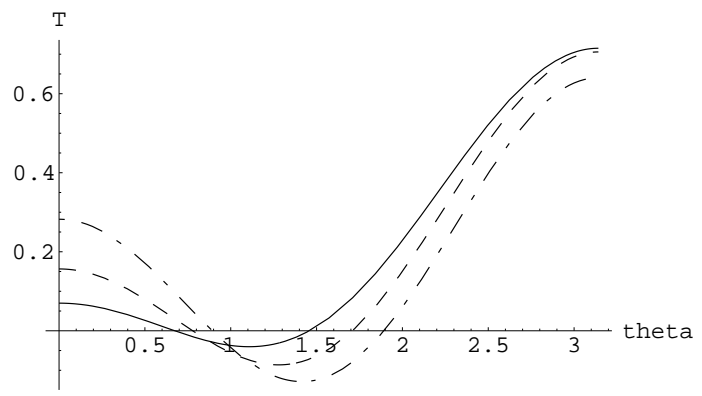

Figure 6: $T(\theta)$ (level $\left.[1,3], J=1, \mathcal{T}_{2}\right) \quad k=100000000$ (solid line), $k=10$ (dashed line), $k=6$ (dot-dashed line) 
(There is also cubic terms $u_{m}^{a j}$ which have coefficients proportional to $k$. This kind of term is generated by the second term of commutation relation (31)). To let the contribution of this term be finite, we rescale $u_{m}^{a j}$ to $\frac{1}{\sqrt{k}} u_{m}^{a j}$. Then the contribution of the linear term in $u_{m}^{a j}$ vanishes. Hence we can get the solution by putting $u_{m}^{a j}$ zero, and its energy is the same as that of level $[0,0]$.

We give the values obtained in level $[1,3]$ analysis to $t_{m}^{j}$ as the initial values and perform the numerical analysis. The results are summarized in the table 1,2 and 3 of Appendix $\mathbb{D}$. In these tables the rescaling of $u_{m}^{a j}$ explained above are explicitly expressed by $\sqrt{k}$ i.e. $u_{m}^{a j}$ in these tables are unrescaled ones.

At large $k$ the value of $R$ is not improved at all, as is expected above. At small $k$ there seems to be small improvement. In the case of $k=4$ and 5 , we do not find any solution corresponding to $\mathcal{T}_{2}^{[1,3]}$. Here we can find a solution corresponding to $\mathcal{T}_{2}^{[2,6]}$ for $k=5$.

\section{5 level $[5 / 2,5]$}

We do not write the full expression of the action because it is not illuminating.

Similarly to the case of level [1,3], we can estimate the value of $R$ at $k \rightarrow \infty$. We rescale the fields as follows so that the most dominant terms in the action give finite contribution.

$$
\begin{aligned}
u^{j j^{\prime}} & \rightarrow \frac{1}{\sqrt{k}} u^{j j^{\prime}}, \\
v^{j j^{\prime}} & \rightarrow \frac{1}{\sqrt{k}} v^{j j^{\prime}}, \\
v^{j j^{\prime} j^{\prime \prime}} & \rightarrow \frac{1}{k} v^{j j^{\prime} j^{\prime \prime}}, \\
\widetilde{u}^{j j^{\prime}} & \rightarrow \frac{1}{\sqrt{k}} \widetilde{u}^{j j^{\prime}} .
\end{aligned}
$$

Then we collect the terms which are finite in the limit $k \rightarrow \infty$ and they can be represented by matrices as has been explained in the previous section. The remaining terms are in fact obtained by considering only the second terms of the commutation relation (3), which is in the same form as that of flat CFT oscillators. Therefore if we put all the components of the string field proportional to a projection operators $P$, the action is equal to $\frac{1}{2 J+1} V \operatorname{tr} P$, where $V$ is the tachyon potential in level $(2,4) . V$ is given in [5] (in the Siegel gauge) and for the vacuum solution it is 0.948553 times the tension of D-brane. Hence we get the lump solutions with $R^{[5 / 2,5]}=0.948553$. 
We look for the sequences of solutions with $R_{n}^{[2,5 / 2]}=0.948553$ at $k \rightarrow \infty$. We take the Siegel gauge to look for the solutions. The results are shown in table 4,5 and 6 of Appendix D. We have checked that the matrices (48) become projections at $k \rightarrow \infty$ for these solutions. The profiles of the solutions are almost the same as those of level [1,3]. Therefore we do not show them.

Now we must check the validity of the Siegel gauge condition [14]. i.e. the equations of motion of $\widetilde{t}^{j}, \widetilde{u}^{j}$ and $\widetilde{u}^{j j^{\prime}}$. We need only the terms linear in $\widetilde{t}^{j}, \widetilde{u}^{j}$ and $\widetilde{u}^{j j^{\prime}}$. See appendix @. Then the equations of motion have linear and quadratic terms in the other components. We see whether the equations of motion for $\widetilde{t}^{j}, \widetilde{u}^{j}$ and $\widetilde{u}^{j j^{\prime}}$ are satisfied when we put the numerical solutions of table. The result is shown in table 7,8 and 9 of Appendix D. For the equations of motion of $\widetilde{t}^{j}$ and $\widetilde{u}^{j}$ linear terms and quadratic terms cancel each other and the ratios of their sums and the linear terms are around 0.2. For the equations of motion of $\widetilde{u}^{j j^{\prime}}$ there seems to be no significant cancellation, but the numerical values themselves are in the same order as those of $\widetilde{t}^{j}$ and $\widetilde{u}^{j}$, or smaller than them. Indeed there are no linear terms in $\widetilde{u}^{j j^{\prime}}$ at $k \rightarrow \infty$ as can be seen by the argument similar to that in the previous section. Therefore each of linear and quadratic terms is small in this limit and they do not have to cancel each other. It seems that these results support the validity of the Siegel gauge condition.

\section{$3.6 k \rightarrow \infty, J=$ fixed}

We saw in the previous discussion that in the case of $J=$ fixed and $k \rightarrow \infty$, the components of the string field form $(2 J+1) \times(2 J+1)$ matrices and the solutions can be constructed by introducing projection operators. The accuracy of the mass of these solution is the same as that of the closed string vacuum. This is true for arbitrary levels. Indeed, the commutation relation is in the same form as that of the flat CFT oscillators in the limit $k \rightarrow \infty$, and $c^{\prime} \rightarrow 23$. (The size of representation matrices of $\mathrm{SU}(2)$ do not get large as $k \rightarrow \infty$ since $J$ is fixed.) Therefore the computation of the coefficients of each terms in the action is completely the same as that of the tachyon potential on the flat CFT. The OPE of primary fields yields the structure of matrix product. As a result the action is equal to the tachyon potential with the components of string field replaced by $(2 J+1) \times(2 J+1)$ matrices. This situation is the same as in the case where constant B-field is present on the flat space [30, 31]. 


\section{Summary and discussions}

We have constructed lump solutions of D-branes on SU(2) group manifold by level truncation approximation. Their energy agree with the expected values with good accuracy. It is expected that the accuracy is improved if we include higher level terms. We have considered the cases $J=\frac{1}{2}, 1$. It is desirable to consider the cases with larger $J$, though the calculation is more cumbersome because the number of primary operators increases. We have seen that in the limit $k \rightarrow \infty, J=$ fixed, the string field can be expressed by matrices, and by taking them to be proportional to a projection matrix the action is written as the tachyon potential times the trace of the projection matrix. This case can also be treated by effective action [20], but our method can be used for investigating the case with small $k$.

The results show the tendency that the accuracy is improved as $k$ increases. One of the reason for this fact is that the dimension of the primary operators decrease and more operators must be included for fixed truncation level. Though for small $k$ we do not always obtain very high accuracy as that of $k \rightarrow \infty$, there seems to be large improvement as we include more terms with higher level. In the case of $k=4, J=1$ we cannot find the solution corresponding to $\mathcal{T}_{2}$. It is expected that we can find the solution if we add higher level terms, as in the case of $k=5$.

At present it is not clear in what sense the level truncation is an approximation. Our results seem to show it is a meaningful approximation in our case.

It is interesting to consider lump solutions of D-branes on $\mathrm{SU}(2)$ group manifold by BSFT. Since the background is curved, it seems to be difficult to obtain exact results as in the case of flat background. One of the difficulty is that we must consider too many operators for satisfying the equations of motion in contrast to the case of flat background, where we can consider only the tachyon field up to quadratic order in coordinates. For the large $k$ case see 32.

Recently the solutions corresponding to D-branes on arbitrary CFT background in vacuum CSFT are constructed in [11]. If the relation between vacuum CSFT and ordinary CSFT becomes clear, we can relate them to our solutions and they must agree.

We have considered the tachyon condensation. But our method can also be applicable to the gluon condensation, by which the opposite phenomena to ours happen i.e. many D0-branes form D2-branes [19]. It is interesting to investigate it by our method. 


\section{Acknowledgments}

I would like to thank H. Hata, S. Shinohara and S. Teraguchi for helpful discussions.

\section{A Quantum 6j-symbol}

The symbol $c_{j_{1}, j_{2}}^{j_{3}}(k, J)$ used in eq.(9) is defined as follows (e.g. [33]).

$$
\begin{aligned}
c_{j_{1}, j_{2}}^{j_{3}}(k, J)= & \left\{\begin{array}{ccc}
j_{1} & j_{2} & j_{3} \\
J & J & J
\end{array}\right\} \\
= & (-1)^{-j_{1}-j_{2}-2 J \sqrt{\left[2 j_{3}+1\right][2 J+1]}} \\
& \times\left[j_{1}\right] !\left[j_{2}\right] !\left[j_{3}\right] ! \\
& \times \sqrt{\frac{\left[2 J-j_{1}\right] !\left[2 J-j_{2}\right] !\left[2 J-j_{3}\right] !}{\left[2 J+j_{1}+1\right] !\left[2 J+j_{2}+1\right] !\left[2 J+j_{3}+1\right] !}} \\
& \times \sqrt{\frac{\left[j_{1}+j_{2}-j_{3}\right] !\left[j_{2}+j_{3}-j_{1}\right] !\left[j_{3}+j_{1}-j_{2}\right] !}{\left[j_{1}+j_{2}+j_{3}+1\right] !}} \\
& \times \sum_{z \geq 0}(-1)^{z}[z+1] !\left\{\left[z-j_{1}-j_{2}-j_{3}\right] !\right. \\
& \times\left[z-j_{1}-2 J\right] !\left[z-j_{2}-2 J\right] !\left[z-j_{3}-2 J\right] !
\end{aligned}
$$

where $\{\cdot\}$ is the quantum $6 \mathrm{j}$-symbol and the sum $\sum_{z \geq 0}$ is only over those $z$ such that the arguments of $[\cdot]$ are nonnegative. $[\cdot]$ is defined as follows.

$$
[n]=\frac{q^{n / 2}-q^{-n / 2}}{q^{1 / 2}-q^{-1 / 2}},
$$

where $q=\exp \left(\frac{2 \pi i}{k+2}\right)$, and

$$
\begin{aligned}
& {[n] !=[1][2] \cdots[n],} \\
& {[0] !=1 .}
\end{aligned}
$$

Note that the above expression of $c_{j_{1}, j_{2}}^{j_{3}}(k, J)$ is totally symmetric under the permutations of $\left(j_{1}, j_{2}, j_{3}\right)$ except the first line. If $k \rightarrow \infty$, then $[n] \rightarrow n$ and the quantum $6 \mathrm{j}$-symbol becomes ordinary $6 \mathrm{j}$-symbol. 


\section{B Cubic terms}

We collect the cubic terms in the action needed for the calculation. These results are applicable to WZW models for general group. We adopt the abbreviation like the last line of eq.(42). For example, $\left\langle 1, J_{-1}^{a}, 1\right\rangle=\frac{2}{3 \sqrt{3}}\left(T_{3}^{a}-T_{1}^{a}\right)=\frac{2}{3 \sqrt{3}}\left\langle\Phi_{j 1}^{m 1^{\prime}}, \Phi_{j 2}^{m 2^{\prime}}, \Phi_{j 3}^{m 3^{\prime}}\right\rangle\left(\delta_{m 1^{\prime}}^{m 1} m_{m 2^{\prime}}^{m 2}\left(T_{\left(j_{3}\right)}^{a}\right)_{m 3^{\prime}}^{m 3}-\right.$ $\left.\left(T_{\left(j_{1}\right)}^{a}\right)_{m 1^{\prime}}^{m 1} \delta_{m 2^{\prime}}^{m 2} \delta_{m 3^{\prime}}^{m 3}\right)$

$$
\begin{aligned}
& \left\langle 1, J_{-1}^{a}, 1\right\rangle=\frac{2}{3 \sqrt{3}}\left(T_{3}^{a}-T_{1}^{a}\right) \\
& \left\langle J_{-1}^{a}, J_{-1}^{b}, 1\right\rangle=-\frac{4}{27}\left[2 T_{1}^{a} T_{1}^{b}-2 T_{2}^{b} T_{2}^{a}+4 T_{1}^{b} T_{2}^{a}+T_{1}^{a} T_{2}^{b}+2 i f^{a b c}\left(T_{1}^{c}-T_{3}^{c}\right)-4 k \delta^{a b}\right] \\
& \left\langle J_{-1}^{a}, J_{-1}^{b}, J_{-1}^{c}\right\rangle=\frac{8}{81 \sqrt{3}}\left[8 k i f^{a b c}+4 k \delta^{a b} T_{1}^{c}-4 k \delta^{c a} T_{1}^{b}\right. \\
& +4 k \delta^{b c} T_{2}^{a}-4 k \delta^{a b} T_{2}^{c}+4 k \delta^{c a} T_{3}^{b}-4 k \delta^{b c} T_{3}^{a} \\
& +4 f^{b a d} f^{c d e} T^{e}-4 f^{b c d} f^{d a e} T_{1}^{e}+4 f^{b a d} f^{c d e} T_{2}^{e} \\
& -4 f^{b c d} f^{d a e} T_{2}^{e}-4 f^{b a d} f^{c d e} T_{3}^{e}+4 f^{b c d} f^{d a e} T_{3}^{e} \\
& { }^{+} 4 i f^{b a d} T_{1}^{c} T_{1}^{d}-4 i f^{c a d} T_{1}^{b} T_{1}^{d}-i f^{b c d} T_{2}^{a} T_{2}^{d}+i f^{b a d} T_{2}^{d} T_{2}^{c}
\end{aligned}
$$

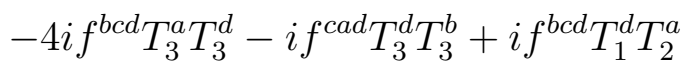

$$
\begin{aligned}
& { }^{-4 i f^{b a d}} T_{1}^{d} T_{2}^{c}-i f^{b a d} T_{1}^{c} T_{2}^{d}+i f^{c a d} T_{1}^{b} T_{2}^{d}-i f^{b c d} T_{1}^{d} T_{3}^{a} \\
& +i f^{b c d} T_{2}^{d} T_{3}^{a}+{ }_{4 i f}^{c a d} T_{1}^{d} T_{3}^{b}+i f^{c a d} T_{2}^{d} T_{3}^{b}+i f^{b a d} T_{1}^{c} T_{3}^{d} \\
& { }_{-i f}^{b a d} T_{2}^{c} T_{3}^{d}+4 i f^{b c d} T_{2}^{a} T_{3}^{d}+i f^{c a d} T_{1}^{b} T_{3}^{d} \\
& +T_{2}^{a} T_{2}^{c} T_{1}^{b}-T_{3}^{a} T_{3}^{b} T_{1}^{c}-T_{1}^{c} T_{1}^{b} T_{2}^{a}+T_{3}^{a} T_{3}^{b} T_{2}^{c} \\
& \left.+T_{1}^{c} T_{1}^{b} T_{3}^{a}-T_{1}^{b} T_{2}^{c} T_{3}^{a}-T_{2}^{a} T_{2}^{c} T_{3}^{b}+T_{1}^{c} T_{2}^{a} T_{3}^{b}\right] \\
& \left\langle 1, J_{-2}^{a}, 1\right\rangle=-\frac{2}{9} T_{2}^{a} \\
& \left\langle 1, J_{-1}^{a} J_{-1}^{b}, 1\right\rangle=\frac{1}{27}\left[4 T_{1}^{b} T_{1}^{a}-4 T_{1}^{b} T_{3}^{a}-4 T_{1}^{a} T_{3}^{b}+4 T_{3}^{b} T_{3}^{a}\right. \\
& \left.-5 i f^{a b c} T_{2}^{c}-5 k \delta^{a b}\right] \\
& \left\langle J_{-1}^{a}, J_{-2}^{b}, 1\right\rangle=\frac{4}{81 \sqrt{3}}\left[3 T_{2}^{b} T_{3}^{a}-3 T_{2}^{a} T_{2}^{b}-16 i f^{a b c} T_{1}^{c}+16 k \delta^{a b}\right] \\
& \left\langle J_{-1}^{a}, J_{-1}^{b} J_{-1}^{c}, 1\right\rangle=\frac{1}{81 \sqrt{3}}\left[32 i k f^{a b c}+10 k \delta^{b c} T_{3}^{a}-10 k \delta^{b c} T_{2}^{a}\right.
\end{aligned}
$$




$$
\begin{aligned}
& +32 k \delta^{a c} T_{3}^{b}+32 k \delta^{a b} T_{3}^{c}-32 k \delta^{a b} T_{1}^{c}-32 k \delta^{a c} T_{1}^{b} \\
& +8 f^{b a d} f^{c d e} T_{3}^{e}-8 f^{b a d} f^{c d e} T_{2}^{e}+32 f^{b a d} f^{c d e} T_{1}^{e} \\
& -8 i f^{b a d} T_{1}^{c} T_{3}^{d}+8 i f^{b a d} T_{1}^{c} T_{2}^{d}+8 i f^{b a d} T_{3}^{d} T_{3}^{c}-8 i f^{b a d} T_{2}^{d} T_{3}^{c} \\
& -8 i f^{c a d} T_{1}^{b} T_{3}^{d}+8 i f^{c a d} T_{1}^{b} T_{2}^{d}-32 i f^{c a d} T_{1}^{d} T_{1}^{b} \\
& -32 i f^{b a d} T_{1}^{c} T_{1}^{d}+32 i f^{b a d} T_{1}^{d} T_{3}^{c}+10 i f^{b c d} T_{2}^{d} T_{3}^{a}-10 i f^{b c d} T_{2}^{a} T_{2}^{d} \\
& { }^{+} 8 i f^{c a d} T_{3}^{d} T_{3}^{b}-8 i f^{c a d} T_{2}^{d} T_{3}^{b}+32 i f^{c a d} T_{1}^{d} T_{3}^{b} \\
& -8 T_{1}^{c} T_{1}^{b} T_{3}^{a}+8 T_{1}^{c} T_{1}^{b} T_{2}^{a}-8 T_{1}^{b} T_{2}^{a} T_{3}^{c}+8 T_{1}^{b} T_{3}^{a} T_{3}^{c} \\
& \left.-8 T_{1}^{c} T_{2}^{a} T_{3}^{b}+8 T_{1}^{c} T_{3}^{a} T_{3}^{b}-8 T_{3}^{a} T_{3}^{c} T_{3}^{b}+8 T_{2}^{a} T_{3}^{c} T_{3}^{b}\right] \\
& \left\langle J_{-2}^{a}, J_{-2}^{b}, 1\right\rangle=\frac{4}{729}\left[-192 k \delta^{a b}-32 i f^{b a c} T_{2}^{c}+32 i f^{b a c} T_{3}^{c}-96 i f^{b a c} T_{1}^{c}+9 T_{1}^{a} T_{2}^{b}\right] \\
& \left\langle J_{-2}^{a}, J_{-1}^{b} J_{-1}^{c}, 1\right\rangle=\frac{1}{729}\left[30 k \delta^{b c} T_{1}^{a}-128 k \delta^{a c} T_{3}^{b}-128 k \delta^{a b} T_{3}^{c}+128 k \delta^{a b} T_{1}^{c}+128 k \delta^{a c} T_{1}^{b}\right. \\
& -384 i k f^{a b c}-296 f^{b a d} f^{c d e} T_{1}^{e}-128 f^{b a d} f^{c d e} T_{3}^{e}+128 f^{b a d} f^{c d e} T_{2}^{e} \\
& -24 i f^{b a d} T_{1}^{d} T_{1}^{c}-40 i f^{b a d} T_{1}^{d} T_{3}^{c}-40 i f^{c a d} T_{1}^{d} T_{1}^{b}
\end{aligned}
$$

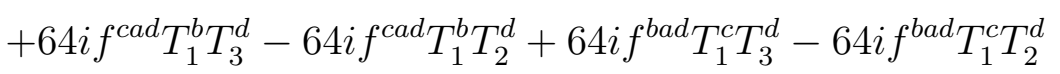

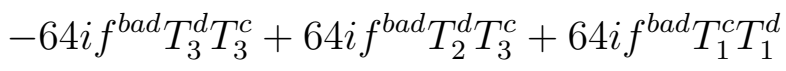

$$
\begin{aligned}
& +30 i f^{b c d} T_{1}^{a} T_{2}^{d}-40 i f^{c a d} T_{1}^{d} T_{3}^{b}-64 i f^{c a d} T_{3}^{d} T_{3}^{b}+64 i f^{c a d} T_{2}^{d} T_{3}^{b} \\
& \left.-24 T_{1}^{a} T_{1}^{c} T_{1}^{b}+24 T_{1}^{a} T_{1}^{b} T_{3}^{c}+24 T_{1}^{a} T_{1}^{c} T_{3}^{b}-24 T_{1}^{a} T_{3}^{c} T_{3}^{b}\right] \\
& \left\langle J_{-1}^{a}, J_{-2}^{b}, J_{-1}^{c}\right\rangle=\frac{8}{729}\left[-12 k \delta^{a c} T_{2}^{b}-16 k \delta^{b c} T_{2}^{a}+16 k \delta^{b c} T_{3}^{a}-16 k \delta^{a b} T_{2}^{c}+16 k \delta^{a b} T_{1}^{c}\right. \\
& +3 i f^{c a d} T_{2}^{b} T_{3}^{d}-3 i f^{c a d} T_{2}^{d} T_{2}^{b}-16 i f^{b a d} T_{1}^{d} T_{2}^{c}+16 i f^{b a d} T_{1}^{c} T_{1}^{d} \\
& { }^{-12 i f^{c a d}} T_{1}^{d} T_{2}^{b}+16 i f^{b c d} T_{3}^{a} T_{3}^{d}-16 i f^{b c d} T_{2}^{a} T_{3}^{d} \\
& \left.-3 T_{1}^{c} T_{2}^{a} T_{2}^{b}+3 T_{1}^{c} T_{2}^{b} T_{3}^{a}+3 T_{2}^{a} T_{2}^{c} T_{2}^{b}-3 T_{2}^{c} T_{2}^{b} T_{3}^{a}\right]
\end{aligned}
$$

Full expressions of $\left\langle J_{-1}^{a}, J_{-1}^{b} J_{-1}^{c}, J_{-1}^{d}\right\rangle$ and $\left\langle J_{-1}^{a} J_{-1}^{b}, J_{-1}^{c} J_{-1}^{d}, 1\right\rangle$ are very long. Therefore we write the expressions which are reduced to some of the above formulae.

$$
\begin{aligned}
\left\langle J_{-1}^{a}, J_{-1}^{b} J_{-1}^{c}, J_{-1}^{d}\right\rangle= & \frac{16}{27} k \delta^{a b}\left\langle 1, J_{-1}^{c}, J_{-1}^{d}\right\rangle+\frac{16}{27} k \delta^{b d}\left\langle J_{-1}^{a}, J_{-1}^{c}, 1\right\rangle \\
& -\frac{5}{27} k \delta^{b c}\left\langle J_{-1}^{a}, 1, J_{-1}^{d}\right\rangle \\
& -\frac{2}{3 \sqrt{3}} i f^{b a e}\left\langle J_{-1}^{e}, J_{-1}^{c}, J_{-1}^{d}\right\rangle+\frac{2}{3 \sqrt{3}} i f^{b d e}\left\langle J_{-1}^{a}, J_{-1}^{c}, J_{-1}^{e}\right\rangle
\end{aligned}
$$




$$
\begin{aligned}
+ & \frac{2}{3 \sqrt{3}}\left\langle J_{-1}^{a}, J_{-1}^{c}, J_{-1}^{d}\right\rangle\left(T_{3}^{b}-T_{1}^{b}\right)+\frac{16}{27} i f^{b a e}\left\langle 1, J_{-1}^{c}, J_{-1}^{d}\right\rangle T_{1}^{e} \\
+ & \frac{16}{27} i f^{b d e}\left\langle J_{-1}^{a}, J_{-1}^{c}, 1\right\rangle T_{3}^{e}-\frac{5}{27} i f^{b c e}\left\langle J_{-1}^{a}, 1, J_{-1}^{d}\right\rangle T_{2}^{e} \\
\left\langle J_{-1}^{a} J_{-1}^{b}, J_{-1}^{c} J_{-1}^{d}, 1\right\rangle= & -\frac{5}{27} k \delta^{c d}\left\langle J_{-1}^{a} J_{-1}^{b}, 1,1\right\rangle+\frac{16}{27} i f^{c a e} f^{e b f}\left\langle J_{-1}^{f}, J_{-1}^{d}, 1\right\rangle \\
& -\frac{2}{3 \sqrt{3}}\left\langle J_{-1}^{a} J_{-1}^{b}, J_{-1}^{d}, 1\right\rangle\left(T_{1}^{c}+T_{3}^{c}\right) \\
& -\frac{2}{3 \sqrt{3}} i f^{c a e}\left\langle J_{-1}^{e} J_{-1}^{b}, J_{-1}^{d}, 1\right\rangle-\frac{2}{3 \sqrt{3}} i f^{c b e}\left\langle J_{-1}^{a} J_{-1}^{e}, J_{-1}^{d}, 1\right\rangle \\
+ & \frac{16}{27} i f^{c a e}\left\langle J_{-1}^{b}, J_{-1}^{d}, 1\right\rangle T_{1}^{e}+\frac{16}{27} i f^{c b e}\left\langle J_{-1}^{a}, J_{-1}^{d}, 1\right\rangle T_{1}^{e} \\
& -\frac{5}{27} i f^{c d e}\left\langle J_{-1}^{a} J_{-1}^{b}, 1,1\right\rangle T_{2}^{e}
\end{aligned}
$$

\section{The Action}

In this appendix we collect the terms necessary for level $[1,3],[2,6]$ and $[5 / 2,5]$ calculations. $S_{0}$ consists of the terms which survive after we take the Siegel gauge condition. $S_{1}$ consists of the linear terms in $\widetilde{t}, \widetilde{u}$ and $\widetilde{u}^{a}$, which are excluded by Siegel gauge condition. $c^{\prime}=26-\frac{3 k}{k+2}$ is the central charge of $X \times Y \times \mathcal{M}$.

$$
\begin{aligned}
& S_{0}=\frac{1}{2}\left(h_{j}-1\right)\langle\Phi, \Phi\rangle t t \\
& +\frac{1}{2}\left(h_{j}\right)\left\langle J_{-1}^{a} \Phi, J_{-1}^{b} \Phi\right\rangle u^{a} u^{b} \\
& +\frac{1}{2}\left(h_{j}+1\right)\left[\left\langle J_{-2}^{a} \Phi, J_{-2}^{b} \Phi\right\rangle v^{a} v^{b}\right. \\
& \left.+2\left\langle J_{-2}^{a} \Phi, J_{-1}^{b} J_{-1}^{c} \Phi\right\rangle v^{a} v^{b c}+\left\langle J_{-1}^{a} J_{-1}^{b} \Phi, J_{-1}^{c} J_{-1}^{d} \Phi\right\rangle v^{a b} v^{c d}-\langle\Phi, \Phi\rangle \beta \beta\right] \\
& +\frac{c^{\prime}}{4}\left(h_{j}+1\right)\langle\Phi, \Phi\rangle w w \\
& +K^{3-h_{j_{1}}-h_{j_{2}}-h_{j_{3}}}\left[\frac{1}{3}\langle\Phi, \Phi, \Phi\rangle t t t+\left\langle\Phi, \Phi, J_{-1}^{a} \Phi\right\rangle t t u^{a}+\left\langle\Phi, J_{-1}^{a} \Phi, J_{-1}^{b} \Phi\right\rangle t u^{a} u^{b}\right. \\
& +\frac{1}{3}\left\langle J_{-1}^{a} \Phi, J_{-1}^{b} \Phi, J_{-1}^{c} \Phi\right\rangle u^{a} u^{b} u^{c}+\left\langle\Phi, \Phi, J_{-2}^{a} \Phi\right\rangle t t v^{a}+\left\langle\Phi, \Phi, J_{-1}^{a} J_{-1}^{b} \Phi\right\rangle t t v^{a b} \\
& +\left\langle\Phi, J_{-1}^{a} \Phi, J_{-2}^{b} \Phi\right\rangle t u^{a} v^{b}+\left\langle\Phi, J_{-2}^{a} \Phi, J_{-1}^{b} \Phi\right\rangle t v^{a} u^{b} \\
& +\left\langle\Phi, J_{-1}^{a} \Phi, J_{-1}^{b} J_{-1}^{c} \Phi\right\rangle t u^{a} v^{b c}+\left\langle\Phi, J_{-1}^{a} J_{-1}^{b} \Phi, J_{-1}^{c} \Phi\right\rangle t v^{a b} u^{c} \\
& +\left\langle J_{-1}^{a} \Phi, J_{-1}^{b} \Phi, J_{-2}^{c} \Phi\right\rangle u^{a} u^{b} v^{c}+\left\langle J_{-1}^{a} \Phi, J_{-1}^{b} \Phi, J_{-1}^{c} J_{-1}^{d} \Phi\right\rangle u^{a} u^{b} v^{c d}
\end{aligned}
$$




$$
\begin{aligned}
& +\left\langle\Phi, J_{-2}^{a} \Phi, J_{-2}^{b} \Phi\right\rangle t v^{a} v^{b} \\
& +\left\langle\Phi, J_{-2}^{a} \Phi, J_{-1}^{b} J_{-1}^{c} \Phi\right\rangle t v^{a} v^{b c}+\left\langle\Phi, J_{-1}^{a} J_{-1}^{b} \Phi, J_{-2}^{c} \Phi\right\rangle t v^{a b} v^{c} \\
& +\left\langle\Phi, J_{-1}^{a} J_{-1}^{b} \Phi, J_{-1}^{c} J_{-1}^{d} \Phi\right\rangle t v^{a b} v^{c d} \\
& -\frac{11}{27}\left\langle\Phi, \Phi, J_{-2}^{a} \Phi\right\rangle t \beta v^{a}-\frac{11}{27}\left\langle\Phi, J_{-2}^{a} \Phi, \Phi\right\rangle t v^{a} \beta \\
& -\frac{11}{27}\left\langle\Phi, \Phi, J_{-1}^{a} J_{-1}^{b} \Phi\right\rangle t \beta v^{a b}-\frac{11}{27}\left\langle\Phi, J_{-1}^{a} J_{-1}^{b} \Phi, \Phi\right\rangle t v^{a b} \beta \\
& -\frac{11}{27}\langle\Phi, \Phi, \Phi\rangle t t \beta-\frac{11}{27}\langle\Phi, \Phi, \Phi\rangle t \beta \beta \\
& -\frac{11}{27}\left\langle\Phi, \Phi, J_{-1}^{a} \Phi\right\rangle t \beta u^{a}-\frac{11}{27}\left\langle\Phi, J_{-1}^{a} \Phi, \Phi\right\rangle t u^{a} \beta \\
& -\frac{11}{27}\left\langle J_{-1}^{a} \Phi, J_{-1}^{b} \Phi, \Phi\right\rangle u^{a} u^{b} \beta \\
& -\frac{5}{54} c^{\prime}\langle\Phi, \Phi, \Phi\rangle t t w+\left(\frac{128}{729} c^{\prime}+\left(\frac{5}{54} c^{\prime}\right)^{2}\right)\langle\Phi, \Phi, \Phi\rangle t w w \\
& -\frac{5}{54} c^{\prime}\left\langle\Phi, J_{-1}^{a} \Phi, \Phi\right\rangle t u^{a} w-\frac{5}{54} c^{\prime}\left\langle\Phi, \Phi, J_{-1}^{a} \Phi\right\rangle t w u^{a} \\
& -\frac{5}{54} c^{\prime}\left\langle J_{-1}^{a} \Phi, J_{-1}^{b} \Phi, \Phi\right\rangle u^{a} u^{b} w \\
& +\frac{55}{1458} c^{\prime}\langle\Phi, \Phi, \Phi\rangle t \beta w+\frac{55}{1458} c^{\prime}\langle\Phi, \Phi, \Phi\rangle t w \beta \\
& -\frac{5}{54} c^{\prime}\left\langle\Phi, J_{-2}^{a} \Phi, \Phi\right\rangle t v^{a} w-\frac{5}{54} c^{\prime}\left\langle\Phi, \Phi, J_{-2}^{a} \Phi\right\rangle t w v^{a} \\
& \left.-\frac{5}{54} c^{\prime}\left\langle\Phi, J_{-1}^{a} J_{-1}^{b} \Phi, \Phi\right\rangle t v^{a b} w-\frac{5}{54} c^{\prime}\left\langle\Phi, \Phi, J_{-1}^{a} J_{-1}^{b} \Phi\right\rangle t w v^{a b}\right] \\
& S_{1}=\langle\Phi, \Phi\rangle \widetilde{t}\left(T^{a} u^{a}\right) \\
& -2\langle\Phi, \Phi\rangle \widetilde{u}\left(T^{a} v^{a}\right)+2\left\langle J_{-1}^{a} \Phi, J_{-1}^{b} \Phi\right\rangle \widetilde{u}^{a} v^{b} \\
& -\left\langle J_{-1}^{a} \Phi, J_{-1}^{b} \Phi\right\rangle \widetilde{u} v^{a b}+\left\langle J_{-1}^{a} \Phi, L_{1} J_{-1}^{b} J_{-1}^{c} \Phi\right\rangle \widetilde{u}^{a} v^{b c} \\
& -3\langle\Phi, \Phi\rangle \widetilde{u} \beta+\langle\Phi, \Phi\rangle \widetilde{u}^{a}\left(T^{a} \beta\right) \\
& -\frac{c^{\prime}}{2}\langle\Phi, \Phi\rangle \tilde{u} w \\
& +\frac{32}{81 \sqrt{3}}\langle\Phi, \Phi, \Phi\rangle t \beta \tilde{t}-\frac{32}{81 \sqrt{3}}\langle\Phi, \Phi, \Phi\rangle \beta t \tilde{t} \\
& +\frac{16}{27}\langle\Phi, \Phi, \Phi\rangle t t \widetilde{u} \\
& +\frac{16}{27}\left\langle\Phi, J_{-1}^{a} \Phi, \Phi\right\rangle t u^{a} \widetilde{u}+\frac{16}{27}\left\langle J_{-1}^{a} \Phi, \Phi, \Phi\right\rangle u^{a} t \widetilde{u} \\
& +\frac{16}{27}\left\langle J_{-1}^{a} \Phi, J_{-1}^{b} \Phi, \Phi\right\rangle u^{a} u^{b} \widetilde{u} \\
& +\frac{16}{27}\left\langle\Phi, J_{-2}^{a} \Phi, \Phi\right\rangle t v^{a} \widetilde{u}+\frac{16}{27}\left\langle J_{-2}^{a} \Phi, \Phi, \Phi\right\rangle v^{a} t \widetilde{u}
\end{aligned}
$$




$$
\begin{aligned}
& +\frac{16}{27}\left\langle\Phi, J_{-1}^{a} J_{-1}^{a} \Phi, \Phi\right\rangle t v^{a b} \widetilde{u}+\frac{16}{27}\left\langle J_{-1}^{a} J_{-1}^{b} \Phi, \Phi, \Phi\right\rangle v^{a b} t \widetilde{u} \\
& +\frac{32}{81 \sqrt{3}}\left\langle J_{-1}^{a} \Phi, \Phi, \Phi\right\rangle u^{a} \beta \widetilde{t}-\frac{32}{81 \sqrt{3}}\left\langle\Phi, J_{-1}^{a} \Phi, \Phi\right\rangle \beta u^{a} \widetilde{t} \\
& +\frac{16}{81}\langle\Phi, \Phi, \Phi\rangle t \beta \widetilde{u}+\frac{16}{81}\langle\Phi, \Phi, \Phi\rangle t \widetilde{u} \beta \\
& +\frac{32}{81 \sqrt{3}}\left\langle\Phi, \Phi, J_{-1}^{a} \Phi\right\rangle t \beta \widetilde{u}^{a}-\frac{32}{81 \sqrt{3}}\left\langle\Phi, J_{-1}^{a} \Phi, \Phi\right\rangle \beta t \widetilde{u}^{a} \\
& \left.-\frac{40}{729} c^{\prime}\langle\Phi, \Phi, \Phi\rangle t \widetilde{u} w-\frac{40}{729} c^{\prime}\langle\Phi, \Phi, \Phi\rangle t w \widetilde{u}\right]
\end{aligned}
$$

\begin{tabular}{|c|c|c|c|c|c|c|}
\hline & $k=4$ & $k=5$ & $k=6$ & $k=7$ & $k=8$ & $k=10$ \\
\hline$t^{0}$ & 0.258107 & 0.27399 & 0.280901 & 0.286079 & 0.289726 & 0.294389 \\
\hline$t^{1}$ & 0.360168 & 0.352909 & 0.349867 & 0.346261 & 0.343223 & 0.338634 \\
\hline$t^{2}$ & -0.158304 & -0.196357 & -0.210897 & -0.221076 & -0.227766 & -0.235527 \\
\hline$\sqrt{k} u^{01}$ & $-9.72804 \times 10^{-11}$ & $-9.55096 \times 10^{-18}$ & $2.08702 \times 10^{-17}$ & $1.83944 \times 10^{-17}$ & $1.08632 \times 10^{-18}$ & $1.40400 \times 10^{-17}$ \\
\hline$\sqrt{k} u^{10}$ & $-2.45936 \times 10^{-9}$ & -0.0216722 & -0.0298519 & -0.0344495 & -0.0372043 & -0.0399092 \\
\hline$\sqrt{k} u^{11}$ & 0 & 0 & 0 & 0 & 0 & 0 \\
\hline$\sqrt{k} u^{12}$ & $-3.45812 \times 10^{-9}$ & -0.0268181 & -0.0337738 & -0.0365391 & -0.0375822 & -0.0376270 \\
\hline$\sqrt{k} u^{21}$ & 0 & 0.0171184 & 0.0249579 & 0.0302724 & 0.0340421 & 0.0387376 \\
\hline$\sqrt{k} u^{22}$ & 0 & 0 & 0 & 0 & 0 & 0 \\
\hline$\sqrt{k} u^{23}$ & 0 & 0.0372721 & 0.0458503 & 0.0494348 & 0.0509581 & 0.0513974 \\
\hline$R_{1}^{\lfloor 2,6]}$ & 0.67064 & 0.656018 & 0.648754 & 0.648206 & 0.649353 & 0.652857 \\
\hline & $k=50$ & $k=1000$ & $\bar{k} k=100000$ & $\bar{k} k=100000000$ & & \\
\hline$t^{0}$ & 0.303815 & 0.304131 & 0.304119 & 0.304119 & & \\
\hline$t^{1}$ & 0.324627 & 0.322641 & 0.322567 & 0.322567 & & \\
\hline$t^{2}$ & -0.243488 & -0.240632 & -0.240429 & -0.240427 & & \\
\hline$\sqrt{k} u^{01}$ & $-4.04713 \times 10^{-17}$ & $-8.12139 \times 10^{-17}$ & $1.79546 \times 10^{-15}$ & $-1.37752 \times 10^{-16}$ & & \\
\hline$\sqrt{k} u^{10}$ & -0.0299926 & -0.00759889 & -0.000764983 & -0.0000241925 & & \\
\hline$\sqrt{k} u^{11}$ & 0 & 0 & 0 & 0 & & \\
\hline$\sqrt{k} u^{12}$ & -0.0225064 & -0.00538937 & -0.000540939 & -0.0000171067 & & \\
\hline$\sqrt{k} u^{21}$ & 0.0351798 & 0.00928736 & 0.000936888 & 0.0000296296 & & \\
\hline$\sqrt{k} u^{22}$ & 0 & 0 & 0 & 0 & & \\
\hline$\sqrt{k} u^{23}$ & 0.0317163 & 0.00762109 & 0.000765006 & 0.0000241925 & & \\
\hline$R_{1}^{\lfloor 2,6]}$ & 0.676028 & 0.684152 & 0.684611 & 0.684616 & & \\
\hline
\end{tabular}

\section{Numerical data}

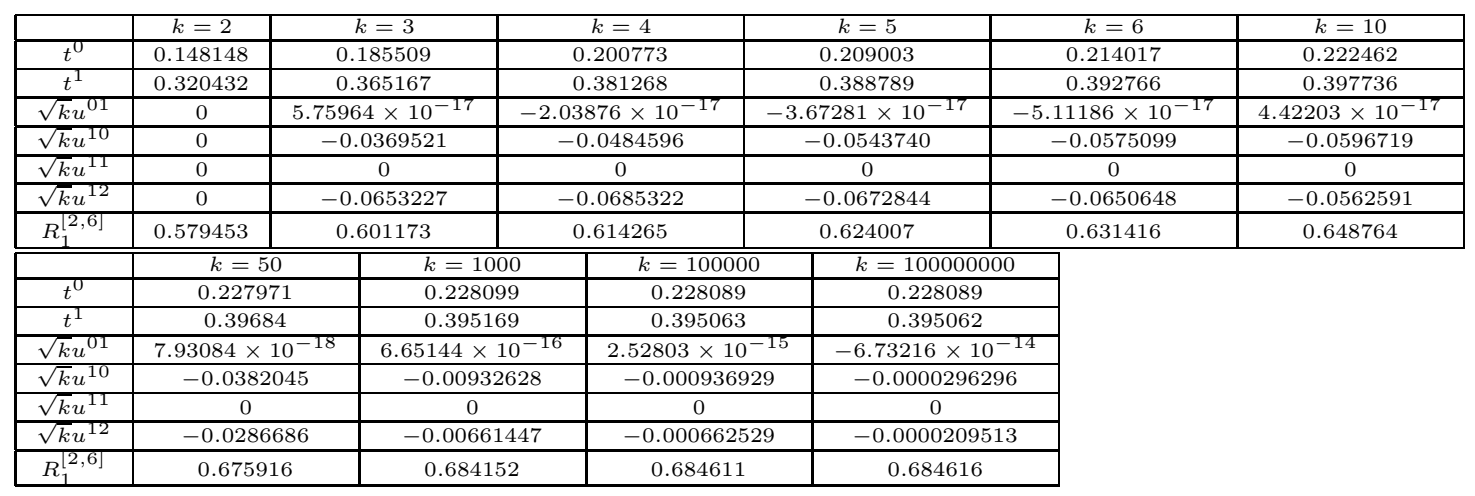

Table 1: $\mathcal{T}_{1}^{[2,6]}$ and $R_{1}^{[2,6]}(J=1 / 2)$

Table 2: $\mathcal{T}_{1}^{[2,6]}$ and $R_{1}^{[2,6]}(J=1)$ 


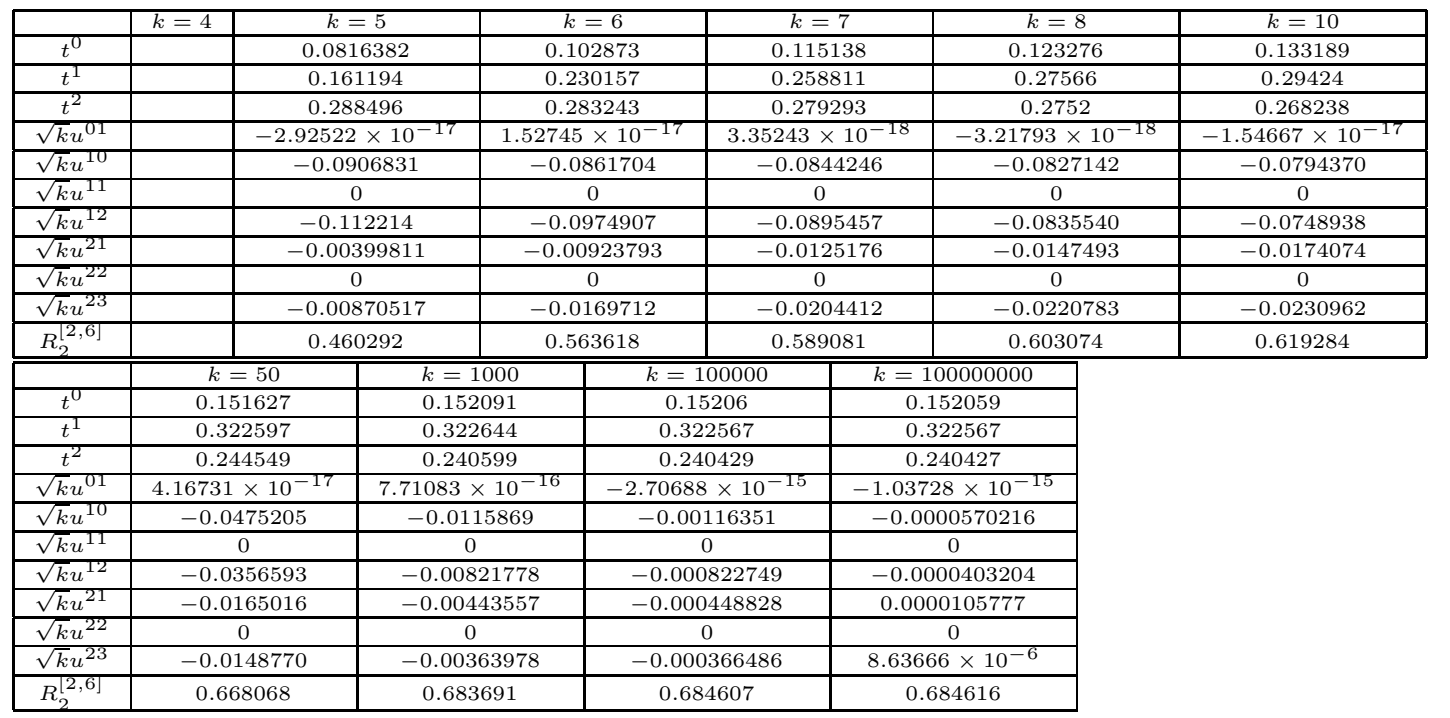

Table 3: $\mathcal{T}_{2}^{[2,6]}$ and $R_{2}^{[2,6]}(J=1)$ 


\begin{tabular}{|c|c|c|c|c|c|c|c|c|c|}
\hline & $k=2$ & $k=3$ & $k=4$ & $k=5$ & $k=6$ & $k=7$ & $k=8$ & $k=9$ & $k=10$ \\
\hline$t^{\sigma}$ & 0.182129 & 0.225759 & 0.242631 & 0.251724 & 0.25716 & 0.260658 & 0.263032 & 0.26471 & 0.265934 \\
\hline$t^{1}$ & 0.36068 & 0.426136 & 0.445349 & 0.454849 & 0.460191 & 0.46345 & 0.465557 & 0.466977 & 0.467967 \\
\hline$\sqrt{k} u^{0,1}$ & 0 & 0.0124587 & 0.00358094 & 0.00187705 & 0.00120394 & 0.00085194 & 0.000638823 & 0.000497805 & 0.000399083 \\
\hline$\sqrt{k} u^{1,0}$ & 0 & -0.0362956 & -0.057079 & -0.0655588 & -0.0697306 & -0.0717213 & -0.0724835 & -0.0725181 & -0.0721097 \\
\hline$\sqrt{k} u^{1,1}$ & 0 & 0 & 0 & 0 & 0 & 0 & 0 & 0 & 0 \\
\hline$\sqrt{k} u^{1,2}$ & 0 & -0.0934329 & -0.0890626 & -0.085463 & -0.0816555 & -0.0780169 & -0.0746705 & -0.0716331 & -0.0688848 \\
\hline$\sqrt{k} v^{0,1}$ & 0 & 0 & 0 & 0 & 0 & 0 & 0 & 0 & 0 \\
\hline$\sqrt{k} v^{1,0}$ & & 0 & 0 & 0 & 0 & 0 & 0 & 0 & 0 \\
\hline$\sqrt{k} v^{1,1}$ & & -0.0416215 & -0.0373648 & -0.0378112 & -0.0383651 & -0.0386618 & -0.0387178 & -0.0385935 & -0.0383426 \\
\hline$\sqrt{k} v^{1,2}$ & & 0 & 0 & 0 & 0 & 0 & 0 & 0 & 0 \\
\hline$k v^{0,1,0}$ & -0.0032832 & -0.00588747 & -0.0101722 & -0.012695 & -0.0144098 & -0.0156477 & -0.016579 & -0.0173019 & -0.0178773 \\
\hline$k v^{0,1,1}$ & 0 & 0 & 0 & 0 & 0 & 0 & 0 & 0 & 0 \\
\hline$k v^{0,1,2}$ & 0.015965 & 0.0106885 & 0.00825364 & 0.0066016 & 0.00549079 & 0.00469881 & 0.00410654 & 0.003647 & 0.00327999 \\
\hline$k v^{1,0,1}$ & & -0.048801 & -0.0385105 & -0.0395883 & -0.0422954 & -0.0452372 & -0.0480562 & -0.0506541 & -0.0530145 \\
\hline$k v^{1,1, \sigma}$ & & 0 & 0 & 0 & 0 & 0 & 0 & 0 & 0 \\
\hline$k v^{1,1,1}$ & & 0.0485517 & 0.038505 & 0.0393737 & 0.0418666 & 0.0446333 & 0.0473165 & 0.0498103 & 0.0520912 \\
\hline$k v^{1,1,2}$ & & 0 & 0 & 0 & 0 & 0 & 0 & 0 & 0 \\
\hline$k v^{1,2,1}$ & & 0.0312945 & 0.0247845 & 0.025381 & 0.0270238 & 0.0288359 & 0.0305873 & 0.032211 & 0.0336933 \\
\hline$k v^{1,2,2}$ & & 0 & 0 & 0 & 0 & 0 & 0 & 0 & 0 \\
\hline$k v^{1,2,3}$ & & 0.00641082 & 0.0067976 & 0.0065752 & 0.00613662 & 0.00567264 & 0.00523985 & 0.0048518 & 0.00450837 \\
\hline$\beta^{0}$ & -0.06644 & -0.0776835 & -0.0825814 & -0.085021 & -0.0863635 & -0.0871489 & -0.0876249 & -0.0879181 & -0.0880984 \\
\hline$\beta^{1}$ & & -0.0609899 & -0.0736911 & -0.083145 & -0.0904823 & -0.0963519 & -0.101159 & -0.10517 & -0.108569 \\
\hline$w^{0}$ & 0.0252397 & 0.0263729 & 0.0274827 & 0.0279216 & 0.0280881 & 0.02813 & 0.028111 & 0.0280617 & 0.0279978 \\
\hline$w^{1}$ & & 0.0186859 & 0.0223821 & 0.025152 & 0.0273088 & 0.0290367 & 0.0304532 & 0.0316362 & 0.0326396 \\
\hline$R_{1}^{[5 / 2,5]}$ & 0.751672 & 0.821284 & 0.838754 & 0.853024 & 0.864079 & 0.872784 & 0.879797 & 0.885567 & 0.890403 \\
\hline
\end{tabular}

\begin{tabular}{|c|c|c|c|c|}
\hline & $k=50$ & $k=1000$ & $k=100000$ & $k=100000000$ \\
\hline$t^{0}$ & 0.27083 & 0.270809 & 0.270796 & 0.270795 \\
\hline$t^{1}$ & 0.470276 & 0.469119 & 0.469032 & 0.469031 \\
\hline$\sqrt{k} u^{0,1}$ & 0.0000116695 & $7.84482 \times 10^{-9}$ & $6.93279 \times 10^{-14}$ & $4.38356 \times 10^{-7}$ \\
\hline$\sqrt{k} u^{1,0}$ & -0.0445576 & -0.0106754 & -0.00107121 & -0.0000334374 \\
\hline$\sqrt{k} u^{1,1}$ & 0 & 0 & 0 & 0 \\
\hline$\sqrt{k} u^{1,2}$ & -0.0334611 & -0.00757131 & -0.000757483 & -0.0000245737 \\
\hline$\sqrt{k} v^{0,1}$ & 0 & 0 & 0 & 0 \\
\hline$\sqrt{k} v^{1,0}$ & 0 & 0 & 0 & 0 \\
\hline$\sqrt{k} v^{1,1}$ & -0.0249031 & -0.00614266 & -0.00061746 & -0.0000195268 \\
\hline$\sqrt{k} v^{1,2}$ & 0 & 0 & 0 & 0 \\
\hline$k v^{0,1,0}$ & -0.0217152 & -0.0224384 & -0.0224725 & -0.0224728 \\
\hline$k v^{0,1,1}$ & 0 & 0 & 0 & 0 \\
\hline$k v^{0,1,2}$ & 0.00064918 & 0.0000321639 & $3.21426 \times 10^{-7}$ & $3.35187 \times 10^{-10}$ \\
\hline$k v^{1,0,1}$ & -0.0801125 & -0.0902368 & -0.0908171 & -0.0908230 \\
\hline$k v^{1,1,0}$ & 0 & 0 & 0 & 0 \\
\hline$k v^{1,1,1}$ & 0.078991 & 0.0892917 & 0.0898854 & 0.0898914 \\
\hline$k v^{1,1,2}$ & 0 & 0 & 0 & 0 \\
\hline$k v^{1,2,1}$ & 0.0510425 & 0.0576411 & 0.0580209 & 0.580248 \\
\hline$k v^{1,2,2}$ & 0 & 0 & 0 & 0 \\
\hline$k v^{1,2,3}$ & 0.00111353 & 0.0000581099 & $5.82313 \times 10^{-7}$ & $5.91053 \times 10^{-10}$ \\
\hline$\beta^{0}$ & -0.0874818 & -0.0866824 & -0.0866323 & -0.0866318 \\
\hline$\beta^{1}$ & -0.139739 & -0.149504 & -0.150045 & -0.150051 \\
\hline$w^{0}$ & 0.0266379 & 0.0259881 & 0.0259497 & 0.0259494 \\
\hline$w^{1}$ & 0.0418775 & 0.0447829 & 0.044944 & 0.0449456 \\
\hline$R a^{5 / 2,5]}$ & 0.93371 & 0.947746 & 0.948545 & 0.948553 \\
\hline
\end{tabular}

Table $4: \mathcal{T}_{1}^{[5 / 2,5]}$ and $R_{1}^{[5 / 2,5]}(J=1 / 2)$ 


\begin{tabular}{|c|c|c|c|c|c|c|c|}
\hline & $k=4$ & $k=5$ & $k=6$ & $k=7$ & $k=8$ & $k=9$ & $k=10$ \\
\hline$t^{0}$ & 0.314788 & 0.331704 & 0.338216 & 0.343281 & 0.346835 & 0.349408 & 0.351321 \\
\hline$t^{1}$ & 0.41735 & 0.409288 & 0.407187 & 0.404102 & 0.401494 & 0.399346 & 0.397583 \\
\hline$t^{2}$ & -0.184485 & -0.225581 & -0.239889 & -0.249824 & -0.256177 & -0.260388 & -0.263254 \\
\hline$\sqrt{k} u^{0,1}$ & $1.02888 \times 10^{-15}$ & 0.000889899 & 0.000605516 & 0.000407051 & 0.000286384 & 0.000210621 & 0.000160798 \\
\hline$\sqrt{k} u^{1,0}$ & $1.08749 \times 10^{-15}$ & -0.0252814 & -0.0350701 & -0.0406004 & -0.0439127 & -0.0459348 & -0.0471537 \\
\hline$\sqrt{k} u^{1,1}$ & 0 & 0 & 0 & 0 & 0 & 0 & 0 \\
\hline$\sqrt{k} u^{1,2}$ & $-1.55042 \times 10^{-15}$ & -0.0338029 & -0.0413802 & -0.0442018 & -0.0451556 & -0.0452445 & -0.0449002 \\
\hline$\sqrt{k} u^{2,1}$ & $-5.77304 \times 10^{-16}$ & 0.0211377 & 0.0308004 & 0.0372146 & 0.0416885 & 0.0448659 & 0.0471382 \\
\hline$\sqrt{k} u^{2,2}$ & 0 & 0 & 0 & 0 & 0 & 0 & 0 \\
\hline$\sqrt{k} u^{2,3}$ & $7.13522 \times 10^{-16}$ & 0.0479757 & 0.0578942 & 0.0616537 & 0.0630256 & 0.0632565 & 0.0628917 \\
\hline$\sqrt{k} v^{0,1}$ & 0 & 0 & 0 & 0 & 0 & 0 & 0 \\
\hline$\sqrt{k} v^{1,0}$ & 0 & 0 & 0 & 0 & 0 & 0 & 0 \\
\hline$\sqrt{k} v^{1,1}$ & -0.0417624 & -0.0362672 & -0.0336249 & -0.0319554 & -0.0307608 & -0.0298178 & -0.0290239 \\
\hline$\sqrt{k} v^{1,2}$ & 0 & 0 & 0 & 0 & 0 & 0 & 0 \\
\hline \multicolumn{8}{|l|}{$\sqrt{k} v^{2,1}$} \\
\hline \multicolumn{8}{|l|}{$\sqrt{k} v^{2,2}$} \\
\hline \multicolumn{8}{|l|}{$\sqrt{k} v^{2,3}$} \\
\hline$k v^{0,1,0}$ & -0.01387752 & -0.0172971 & -0.0194408 & -0.021064 & -0.022311 & -0.0232982 & -0.0240986 \\
\hline$k v^{0,1,1}$ & 0 & 0 & 0 & 0 & 0 & 0 & 0 \\
\hline$k v^{0,1,2}$ & 0.00899444 & 0.00757175 & 0.00645066 & 0.00563377 & 0.00500638 & 0.00450982 & 0.00410681 \\
\hline$k v^{1,0,1}$ & -0.068786 & -0.0607325 & -0.0568655 & -0.0548976 & -0.0539287 & -0.0534971 & -0.0533688 \\
\hline$k v^{1,1,0}$ & 0 & 0 & 0 & 0 & 0 & 0 & 0 \\
\hline$k v^{1,1,1}$ & 0.069336 & 0.0623375 & 0.0586612 & 0.0566855 & 0.0556311 & 0.0550894 & 0.0548466 \\
\hline$k v^{1,1,2}$ & 0 & 0 & 0 & 0 & 0 & 0 & 0 \\
\hline$k v^{1,2,1}$ & 0.0445292 & 0.0398361 & 0.0374356 & 0.0361654 & 0.0355021 & 0.0351731 & 0.0350373 \\
\hline$k v^{1,2,2}$ & 0 & 0 & 0 & 0 & 0 & 0 & 0 \\
\hline$k v^{1,2,3}$ & -0.0123896 & -0.0088196 & -0.00622704 & -0.00462895 & -0.00357675 & -0.0028504 & -0.00232872 \\
\hline \multicolumn{8}{|l|}{$k v^{2,1,0}$} \\
\hline \multicolumn{8}{|l|}{$k v^{2,1,1}$} \\
\hline \multicolumn{8}{|l|}{$k v^{2,1,2}$} \\
\hline \multicolumn{8}{|l|}{$k v^{2,2,1}$} \\
\hline \multicolumn{8}{|l|}{$k v^{2,2,2}$} \\
\hline \multicolumn{8}{|l|}{$k v^{2,2,3}$} \\
\hline \multicolumn{8}{|l|}{$k v^{2,3,2}$} \\
\hline \multicolumn{8}{|l|}{$k v^{2,3,3}$} \\
\hline \multicolumn{8}{|l|}{$k v^{2,3,4}$} \\
\hline$\beta^{0}$ & -0.105789 & -0.110894 & -0.112718 & -0.114132 & -0.115101 & -0.115783 & -0.116275 \\
\hline$\beta^{1}$ & -0.0669474 & -0.0721441 & -0.0770841 & -0.0807792 & -0.0837999 & -0.0863316 & -0.0884924 \\
\hline \multicolumn{8}{|l|}{$\beta^{2}$} \\
\hline$w^{0}$ & 0.0346485 & 0.0359941 & 0.0363952 & 0.0367119 & 0.0369176 & 0.0370535 & 0.0371441 \\
\hline$w^{1}$ & 0.0188242 & 0.0199966 & 0.0212771 & 0.0222087 & 0.0229717 & 0.0236128 & 0.0241617 \\
\hline \multicolumn{8}{|l|}{$w^{2}$} \\
\hline$R_{1}^{[5 / 2,5]}$ & 0.933395 & 0.908232 & 0.894435 & 0.891439 & 0.891457 & 0.892623 & 0.894206 \\
\hline
\end{tabular}




\begin{tabular}{|c|c|c|c|c|c|c|c|}
\hline & $k=12$ & $k=14$ & $k=17$ & $k=50$ & $k=1000$ & $k=100000$ & $k=100000000$ \\
\hline$t^{0}$ & 0.355306 & 0.357005 & 0.358529 & 0.361078 & 0.361079 & 0.361061 & 0.361061 \\
\hline$t^{1}$ & 0.393787 & 0.391735 & 0.389663 & 0.384585 & 0.383032 & 0.382963 & 0.382962 \\
\hline$t^{2}$ & -0.278162 & -0.281215 & -0.283798 & -0.286995 & -0.285579 & -0.285445 & -0.285443 \\
\hline$\sqrt{k} u^{0,1}$ & 0.000138589 & 0.000111494 & 0.0000830053 & 0.0000109995 & $9.38782 \times 10^{-9}$ & $1.00141 \times 10^{-13}$ & $2.95267 \times 10^{-8}$ \\
\hline$\sqrt{k} u^{1,0}$ & -0.0493389 & -0.0493019 & -0.0483803 & -0.0353295 & -0.00870582 & -0.000874628 & -0.0000276233 \\
\hline$\sqrt{k} u^{1,1}$ & 0 & 0 & 0 & 0 & 0 & 0 & 0 \\
\hline$\sqrt{k} u^{1,2}$ & -0.0447839 & -0.0432117 & -0.0408498 & -0.0265403 & -0.00617445 & -0.000618474 & -0.0000196093 \\
\hline$\sqrt{k} u^{2,1}$ & 0.0515573 & 0.0528416 & 0.0532301 & 0.0418617 & 0.0106457 & 0.00107118 & 0.0000338463 \\
\hline$\sqrt{k} u^{2,2}$ & 0 & 0 & 0 & 0 & 0 & 0 & 0 \\
\hline$\sqrt{k} u^{2,3}$ & 0.0634499 & 0.0613673 & 0.0581311 & 0.0377635 & 0.00873577 & 0.000874658 & 0.0000276956 \\
\hline$\sqrt{k} v^{0,1}$ & 0 & 0 & 0 & 0 & 0 & 0 & 0 \\
\hline$\sqrt{k} v^{1,0}$ & 0 & 0 & 0 & 0 & 0 & 0 & 0 \\
\hline$\sqrt{k} v^{1,1}$ & -0.0304986 & -0.0295192 & -0.0282152 & -0.019975 & -0.00500974 & -0.000504147 & -0.0000159482 \\
\hline$\sqrt{k} v^{1,2}$ & 0 & 0 & 0 & 0 & 0 & 0 & 0 \\
\hline$\sqrt{k} v^{2,1}$ & 0 & 0 & 0 & 0 & 0 & 0 & 0 \\
\hline$\sqrt{k} v^{2,2}$ & 0.0197379 & 0.0215403 & 0.0231784 & 0.0224966 & 0.00642606 & 0.000650809 & 0.0000205775 \\
\hline$\sqrt{k} v^{2,3}$ & 0 & 0 & 0 & 0 & 0 & 0 & 0 \\
\hline$k v^{0,1,0}$ & -0.0247824 & -0.0255961 & -0.0264488 & -0.0289154 & -0.0299178 & -0.0299633 & -0.0299637 \\
\hline$k v^{0,1,1}$ & 0 & 0 & 0 & 0 & 0 & 0 & 0 \\
\hline$k v^{0,1,2}$ & 0.00345395 & 0.00298358 & 0.00247767 & 0.000860433 & 0.0000428906 & $4.28569 \times 10^{-7}$ & $4.28666 \times 10^{-10}$ \\
\hline$k v^{1,0,1}$ & -0.0561326 & -0.0570219 & -0.058401 & -0.0665359 & -0.0736984 & -0.0741519 & -0.0741946 \\
\hline$k v^{1,1,0}$ & 0 & 0 & 0 & 0 & 0 & 0 & 0 \\
\hline$k v^{1,1,1}$ & 0.0574638 & 0.0581291 & 0.0592386 & 0.0663568 & 0.0729662 & 0.0733916 & 0.0734289 \\
\hline$k v^{1,1,2}$ & 0 & 0 & 0 & 0 & 0 & 0 & 0 \\
\hline$k v^{1,2,1}$ & 0.0367481 & 0.0372163 & 0.0379787 & 0.0427422 & 0.0470952 & 0.0473741 & 0.0474194 \\
\hline$k v^{1,2,2}$ & 0 & 0 & 0 & 0 & 0 & 0 & 0 \\
\hline$k v^{1,2,3}$ & -0.00117633 & -0.000764075 & -0.000410992 & 0.000105204 & 0.0000119647 & $1.23327 \times 10^{-7}$ & $1.25443 \times 10^{-10}$ \\
\hline$k v^{2,1,0}$ & -0.00106331 & -0.00127834 & -0.00143850 & -0.00108355 & -0.0000752970 & $-7.65221 \times 10^{-7}$ & $-7.62379 \times 10^{-10}$ \\
\hline$k v^{2,1,1}$ & 0 & 0 & 0 & 0 & 0 & 0 & 0 \\
\hline$k v^{2,1,2}$ & 0.0185435 & 0.0249567 & 0.0332872 & 0.0730424 & 0.104021 & 0.105919 & 0.105885 \\
\hline$k v^{2,2,1}$ & 0 & 0 & 0 & 0 & 0 & 0 & 0 \\
\hline$k v^{2,2,2}$ & -0.00888098 & -0.0122799 & -0.0166614 & -0.0374381 & -0.0536953 & -0.0546959 & -0.0546833 \\
\hline$k v^{2,2,3}$ & 0 & 0 & 0 & 0 & 0 & 0 & 0 \\
\hline$k v^{2,3,2}$ & -0.0137015 & -0.0186574 & -0.0250729 & -0.0556006 & -0.0794333 & -0.0808965 & -0.0808575 \\
\hline$k v^{2,3,3}$ & 0 & 0 & 0 & 0 & 0 & 0 & 0 \\
\hline$k v^{2,3,4}$ & -0.00632142 & -0.00589583 & -0.00527583 & -0.00223821 & -0.000122223 & $-1.22723 \times 10^{-6}$ & $-1.23027 \times 10^{-9}$ \\
\hline$\beta^{0}$ & -0.116649 & -0.116923 & -0.11709 & -0.116564 & -0.115576 & -0.115510 & -0.115509 \\
\hline$\beta^{1}$ & -0.0934840 & -0.0964424 & -0.0998803 & -0.113246 & -0.122002 & -0.122511 & -0.122516 \\
\hline$\beta^{2}$ & 0.0378906 & 0.043247 & 0.0495507 & 0.0742366 & 0.0903689 & 0.0913084 & 0.0913180 \\
\hline$w^{0}$ & 0.0365577 & 0.0364844 & 0.0363524 & 0.0354623 & 0.0346507 & 0.0345997 & 0.0345991 \\
\hline$w^{1}$ & 0.0267857 & 0.0277387 & 0.0288614 & 0.0333927 & 0.0365112 & 0.0366961 & 0.0366979 \\
\hline$w^{2}$ & -0.0119448 & -0.0135931 & -0.0154986 & -0.0226491 & -0.0270963 & -0.0273504 & -0.0273530 \\
\hline$R_{1}^{[5 / 2,5]}$ & 0.904861 & 0.908966 & 0.913995 & 0.934266 & 0.947748 & 0.948545 & 0.948553 \\
\hline
\end{tabular}

Table 5: $\mathcal{T}_{1}^{[5 / 2,5]}$ and $R_{1}^{[5 / 2,5]}(J=1)$ 


\begin{tabular}{|c|c|c|c|c|c|c|c|}
\hline & $k=4$ & $k=5$ & $k=6$ & $k=7$ & $k=8$ & $k=9$ & $k=10$ \\
\hline$t^{0}$ & & 0.108574 & 0.130853 & 0.143935 & 0.152574 & 0.158614 & 0.163012 \\
\hline$t^{1}$ & & 0.216655 & 0.282623 & 0.311856 & 0.329479 & 0.341154 & 0.34935 \\
\hline$t^{2}$ & & 0.306499 & 0.30633 & 0.305413 & 0.303459 & 0.301319 & 0.299306 \\
\hline$\sqrt{k} u^{0,1}$ & & -0.0000180229 & 0.000129681 & 0.000184364 & 0.000191436 & 0.000180074 & 0.000162688 \\
\hline$\sqrt{k} u^{1,0}$ & & -0.114929 & -0.108674 & -0.106245 & -0.103925 & -0.101672 & -0.0994938 \\
\hline$\sqrt{k} u^{1,1}$ & & 0 & 0 & 0 & 0 & 0 & 0 \\
\hline$\sqrt{k} u^{1,2}$ & & -0.142204 & -0.123077 & -0.112891 & -0.105201 & -0.0990687 & -0.0940038 \\
\hline$\sqrt{k} u^{2,1}$ & & -0.00429864 & -0.0104174 & -0.0143277 & -0.0170257 & -0.0189278 & -0.0202891 \\
\hline$\sqrt{k} u^{2,2}$ & & 0 & 0 & 0 & 0 & 0 & 0 \\
\hline$\sqrt{k} u^{2,3}$ & & -0.00933469 & -0.0193081 & -0.0236304 & -0.0257211 & -0.0267095 & -0.0271114 \\
\hline$\sqrt{k} v^{0,1}$ & & 0 & 0 & 0 & 0 & 0 & 0 \\
\hline$\sqrt{k} v^{1,0}$ & & 0 & 0 & 0 & 0 & 0 & 0 \\
\hline$\sqrt{k} v^{1,1}$ & & -0.0166421 & -0.0234621 & -0.0269938 & -0.0291172 & -0.030423 & -0.0312168 \\
\hline$\sqrt{k} v^{1,2}$ & & 0 & 0 & 0 & 0 & 0 & 0 \\
\hline$\sqrt{k} v^{2,1}$ & & & & & & & \\
\hline \multicolumn{5}{|l|}{$\sqrt{k} v^{2,2}$} & & & \\
\hline \multicolumn{8}{|l|}{$\sqrt{k} v^{2,3}$} \\
\hline$k v^{0,1,0}$ & & 0.00174226 & -0.00215824 & -0.00455361 & -0.00627619 & -0.00757253 & -0.00858088 \\
\hline$k v^{0,1,1}$ & & 0 & 0 & 0 & 0 & 0 & 0 \\
\hline$k v^{0,1,2}$ & & 0.0119636 & 0.00937116 & 0.00786394 & 0.00678458 & 0.00597277 & 0.00533992 \\
\hline$k v^{1,0,1}$ & & -0.0136868 & -0.0208768 & -0.0261862 & -0.030628 & -0.0344043 & -0.0376524 \\
\hline$k v^{1,1,0}$ & & 0 & 0 & 0 & 0 & 0 & 0 \\
\hline$k v^{1,1,1}$ & & 0.0116287 & 0.0186019 & 0.0239239 & 0.0283927 & 0.0321994 & 0.0354798 \\
\hline$k v^{1,1, z}$ & & 0 & 0 & 0 & 0 & 0 & 0 \\
\hline$k v^{1,2,1}$ & & 0.00785285 & 0.0123799 & 0.0158031 & 0.0186746 & 0.0211193 & 0.023225 \\
\hline$k v^{1,2,2}$ & & 0 & 0 & 0 & 0 & 0 & 0 \\
\hline$k v^{1,2,3}$ & & 0.00730255 & 0.00831474 & 0.00805819 & 0.00756586 & 0.00704831 & 0.00656395 \\
\hline \multicolumn{8}{|l|}{$k v^{2,1,0}$} \\
\hline \multicolumn{8}{|l|}{$k v^{2,1,1}$} \\
\hline \multicolumn{8}{|l|}{$k v^{2,1,2}$} \\
\hline \multicolumn{8}{|l|}{$k v^{2,2,1}$} \\
\hline \multicolumn{8}{|l|}{$k v^{2,2,2}$} \\
\hline \multicolumn{8}{|l|}{$k v^{2,2,3}$} \\
\hline \multicolumn{8}{|l|}{$k v^{2,3,2}$} \\
\hline \multicolumn{8}{|l|}{$k v^{2,3,3}$} \\
\hline \multicolumn{8}{|l|}{$k v^{2,3,4}$} \\
\hline$\beta^{0}$ & & -0.0399187 & -0.0468744 & -0.0508617 & -0.0534163 & -0.0551511 & -0.0563783 \\
\hline$\beta^{1}$ & & -0.0428108 & -0.0593731 & -0.0688178 & -0.0756665 & -0.0809683 & -0.0852303 \\
\hline \multicolumn{8}{|l|}{$\beta^{2}$} \\
\hline$w^{0}$ & & 0.0152634 & 0.0170702 & 0.0180734 & 0.0186733 & 0.0190507 & 0.0192958 \\
\hline$w^{1}$ & & 0.0147015 & 0.0199323 & 0.0227881 & 0.0248318 & 0.0264046 & 0.0276651 \\
\hline \multicolumn{8}{|l|}{$w^{2}$} \\
\hline$R_{2}^{[5 / 2,5]}$ & & 0.697985 & 0.787555 & 0.809146 & 0.822861 & 0.833042 & 0.841123 \\
\hline
\end{tabular}




\begin{tabular}{|c|c|c|c|c|c|c|c|}
\hline & $k=12$ & $k=14$ & $k=17$ & $k=50$ & $k=1000$ & $k=100000$ & $k=100000000$ \\
\hline$t^{0}$ & 0.169426 & 0.172746 & 0.175698 & 0.180675 & 0.180581 & 0.180531 & 0.180530 \\
\hline$t^{1}$ & 0.361366 & 0.367315 & 0.372586 & 0.382136 & 0.382989 & 0.382963 & 0.382962 \\
\hline$t^{2}$ & 0.295594 & 0.293978 & 0.292218 & 0.287398 & 0.285544 & 0.285444 & 0.285443 \\
\hline$\sqrt{k} u^{0,1}$ & 0.000220801 & 0.000176212 & 0.000128004 & 0.0000149582 & $1.19311 \times 10^{-8}$ & $1.12789 \times 10^{-13}$ & $-1.44719 \times 10^{-8}$ \\
\hline$\sqrt{k} u^{1,0}$ & -0.100828 & -0.0967899 & -0.0914015 & -0.0606565 & -0.0144187 & -0.00144609 & -0.0000457406 \\
\hline$\sqrt{k} u^{1,1}$ & 0 & 0 & 0 & 0 & 0 & 0 & 0 \\
\hline$\sqrt{k} u^{1,2}$ & -0.0910103 & -0.0844522 & -0.0769076 & -0.0455348 & -0.0102262 & -0.00102257 & -0.0000323221 \\
\hline$\sqrt{k} u^{2,1}$ & -0.0174166 & -0.0180781 & -0.0183917 & -0.0146409 & -0.00369371 & -0.000371329 & -0.0000117476 \\
\hline$\sqrt{k} u^{2,2}$ & 0 & 0 & 0 & 0 & 0 & 0 & 0 \\
\hline$\sqrt{k} u^{2,3}$ & -0.0216297 & -0.0211472 & -0.0201924 & -0.0132189 & -0.00303102 & -0.000303204 & $-9.58324 \times 10^{-6}$ \\
\hline$\sqrt{k} v^{0,1}$ & 0 & 0 & 0 & 0 & 0 & 0 & 0 \\
\hline$\sqrt{k} v^{1,0}$ & 0 & 0 & 0 & 0 & 0 & 0 & 0 \\
\hline$\sqrt{k} v^{1,1}$ & -0.0289082 & -0.0288156 & -0.0282087 & -0.0204471 & -0.00501921 & -0.000504157 & -0.0000159397 \\
\hline$\sqrt{k} v^{1,2}$ & 0 & 0 & 0 & 0 & 0 & 0 & 0 \\
\hline$\sqrt{k} v^{2,1}$ & 0 & 0 & 0 & 0 & 0 & 0 & 0 \\
\hline$\sqrt{k} v^{2,2}$ & -0.024225 & -0.0251935 & -0.0259052 & -0.0228624 & -0.00642731 & -0.000650811 & -0.0000205863 \\
\hline$\sqrt{k} v^{2,3}$ & 0 & 0 & 0 & 0 & 0 & 0 & 0 \\
\hline$k v^{0,1,0}$ & -0.0093589 & -0.0103545 & -0.0113574 & -0.0140093 & -0.0149428 & -0.0149815 & -0.0149819 \\
\hline$k v^{0,1,1}$ & 0 & 0 & 0 & 0 & 0 & 0 & 0 \\
\hline$k v^{0,1,2}$ & 0.00466621 & 0.00396889 & 0.00324558 & 0.00108347 & 0.0000532027 & $5.31205 \times 10^{-7}$ & $5.31116 \times 10^{-10}$ \\
\hline$k v^{1,0,1}$ & -0.0414168 & -0.0451461 & -0.0494101 & -0.0648339 & -0.0736724 & -0.0741517 & -0.0741255 \\
\hline$k v^{1,1,0}$ & 0 & 0 & 0 & 0 & 0 & 0 & 0 \\
\hline$k v^{1,1,1}$ & 0.0389862 & 0.0428163 & 0.0472180 & 0.0633867 & 0.0728707 & 0.0733907 & 0.0733690 \\
\hline$k v^{1,1,2}$ & 0 & 0 & 0 & 0 & 0 & 0 & 0 \\
\hline$k v^{1,2,1}$ & 0.025528 & 0.0279752 & 0.0307837 & 0.0410573 & 0.0470461 & 0.0473736 & 0.0473422 \\
\hline$k v^{1,2,2}$ & 0 & 0 & 0 & 0 & 0 & 0 & 0 \\
\hline$k v^{1,2,3}$ & 0.0053121 & 0.0046877 & 0.0039728 & 0.00145488 & 0.0000742872 & $7.43245 \times 10^{-7}$ & $7.42587 \times 10^{-10}$ \\
\hline$k v^{2,1,0}$ & -0.00459930 & -0.00424815 & -0.00380153 & -0.00172786 & -0.000101270 & $-1.02111 \times 10^{-6}$ & $-1.02082 \times 10^{-9}$ \\
\hline$k v^{2,1,1}$ & 0 & 0 & 0 & 0 & 0 & 0 & 0 \\
\hline$k v^{2,1,2}$ & -0.0334439 & -0.0390379 & -0.0459434 & -0.0779293 & -0.104242 & -0.105921 & -0.105969 \\
\hline$k v^{2,2,1}$ & 0 & 0 & 0 & 0 & 0 & 0 & 0 \\
\hline$k v^{2,2,2}$ & 0.0107035 & 0.0140437 & 0.0181942 & 0.0376032 & 0.0536692 & 0.0546957 & 0.0547196 \\
\hline$k v^{2,2,3}$ & 0 & 0 & 0 & 0 & 0 & 0 & 0 \\
\hline$k v^{2,3,2}$ & 0.0211825 & 0.0257547 & 0.0314171 & 0.0577668 & 0.0795089 & 0.0808974 & 0.0809434 \\
\hline$k v^{2,3,3}$ & 0 & 0 & 0 & 0 & 0 & 0 & 0 \\
\hline$k v^{2,3,4}$ & 0.00118433 & 0.00113442 & 0.00104673 & 0.00049862 & 0.0000293805 & $2.96340 \times 10^{-7}$ & $2.96127 \times 10^{-10}$ \\
\hline$\beta^{0}$ & -0.0575724 & -0.0583195 & -0.0588883 & -0.0589482 & -0.0578371 & -0.0577554 & -0.0577546 \\
\hline$\beta^{1}$ & -0.090402 & -0.0947988 & -0.0995262 & -0.114572 & -0.122119 & -0.122512 & -0.122519 \\
\hline$\beta^{2}$ & -0.0379856 & -0.0429174 & -0.0487671 & -0.072916 & -0.0902554 & -0.0913073 & -0.0913180 \\
\hline$w^{0}$ & 0.0191403 & 0.0191689 & 0.0191142 & 0.0182665 & 0.0173586 & 0.0173002 & 0.0172996 \\
\hline$w^{1}$ & 0.0285543 & 0.029739 & 0.0309979 & 0.0348483 & 0.0366107 & 0.0366971 & 0.0366979 \\
\hline$w^{2}$ & 0.0103462 & 0.0119149 & 0.0137721 & 0.0214459 & 0.0270102 & 0.0273496 & 0.0273530 \\
\hline$R_{2}^{[5 / 2,5]}$ & 0.861307 & 0.870218 & 0.88037 & 0.919601 & 0.946874 & 0.948536 & 0.948553 \\
\hline
\end{tabular}

Table $6: \mathcal{T}_{2}^{[5 / 2,5]}$ and $R_{2}^{[5 / 2,5]}(J=1)$ 


\begin{tabular}{|c|c|c|c|c|c|c|c|c|c|}
\hline & & $k=2$ & $k=3$ & $k=4$ & $k=5$ & \multicolumn{2}{|c|}{$k=6$} & $k=7$ & $k=8$ \\
\hline \multirow[t]{2}{*}{$\widetilde{t^{0}}$} & linear & 0 & 0 & 0 & 0 & \multicolumn{2}{|c|}{0} & 0 & 0 \\
\hline & quadratic & 0 & 0 & 0 & 0 & \multicolumn{2}{|c|}{0} & 0 & 0 \\
\hline \multirow[t]{2}{*}{$\widetilde{t^{1}}$} & linear & 0 & 0 & 0 & 0 & \multicolumn{2}{|c|}{0} & 0 & 0 \\
\hline & quadratic & 0 & 0 & 0 & 0 & \multicolumn{2}{|c|}{0} & 0 & 0 \\
\hline \multirow[t]{4}{*}{$\widetilde{u^{0}}$} & linear & -0.115553 & -0.096259 & -0.0996669 & -0.0999901 & \multicolumn{2}{|c|}{-0.0994142} & -0.0985275 & -0.0975507 \\
\hline & quadratic & 0.0801271 & 0.0760034 & 0.0774179 & 0.0773437 & \multicolumn{2}{|c|}{0.0767866} & 0.0760797 & 0.075348 \\
\hline & sum & -0.0354259 & -0.0202556 & -0.022249 & -0.0226464 & \multirow{2}{*}{\multicolumn{2}{|c|}{$\begin{array}{c}-0.0226276 \\
0.227609\end{array}$}} & -0.0224478 & -0.0222027 \\
\hline & sum/linear & 0.306577 & 0.210428 & 0.223234 & 0.226486 & & & 0.227833 & 0.227602 \\
\hline \multirow[t]{4}{*}{$u^{1}$} & linear & & -0.0449295 & -0.0409692 & -0.040177 & \multicolumn{2}{|c|}{-0.0399862} & -0.0399995 & -0.040098 \\
\hline & quadratic & & 0.0328215 & 0.0327684 & 0.032912 & \multirow{2}{*}{\multicolumn{2}{|c|}{$\begin{array}{c}0.0330957 \\
-0.0068905\end{array}$}} & 0.0332766 & 0.0334437 \\
\hline & sum & & -0.012108 & -0.0082008 & -0.007265 & & & -0.0067229 & -0.0066543 \\
\hline & sum/linear & & 0.269489 & 0.20017 & 0.180825 & \multicolumn{2}{|c|}{0.172322} & 0.168075 & 0.165951 \\
\hline \multirow{2}{*}{$\sqrt{k} u^{0,0}$} & linear & 0 & 0 & 0 & 0 & \multicolumn{2}{|c|}{0} & 0 & 0 \\
\hline & quadratic & 0 & 0 & 0 & 0 & \multicolumn{2}{|c|}{0} & 0 & 0 \\
\hline \multirow[t]{2}{*}{$\sqrt{k} u^{1,0}$} & linear & & 0 & 0 & 0 & \multicolumn{2}{|c|}{0} & 0 & 0 \\
\hline & quadratic & & 0 & 0 & 0 & \multicolumn{2}{|c|}{0} & 0 & 0 \\
\hline \multirow{2}{*}{$\sqrt{k} u^{1,1}$} & linear & & 0.00395757 & 0.00316496 & 0.00326707 & \multicolumn{2}{|c|}{0.00337508} & 0.00344044 & 0.00347031 \\
\hline & quadratic & & -0.00223712 & -0.00176369 & -0.00143251 & -0 . & 011936 & -0.00101502 & -0.000877389 \\
\hline$\sqrt{k} u^{1,2}$ & linear & & 0 & 0 & 0 & & 0 & 0 & 0 \\
\hline & quadratic & & 0 & 0 & 0 & & 0 & 0 & 0 \\
\hline & & $k=9$ & $k=10$ & $k=50$ & $\bar{k} k=10$ & & & $=100000$ & $k=100000000$ \\
\hline$\widetilde{t^{0}}$ & linear & 0 & 0 & 0 & 0 & & & 0 & 0 \\
\hline & quadratic & 0 & 0 & 0 & 0 & & & 0 & 0 \\
\hline$\widetilde{t^{1}}$ & linear & 0 & 0 & 0 & 0 & & & 0 & 0 \\
\hline & quadratic & 0 & 0 & 0 & 0 & & & 0 & 0 \\
\hline$\widetilde{u^{0}}$ & linear & -0.0965762 & -0.0956433 & -0.0830391 & -0.0777 & & & 0774489 & -0.0774462 \\
\hline & quadratic & 0.0746406 & 0.0739763 & 0.065545 & 0.06217 & & & $\overline{1619843}$ & 0.0619821 \\
\hline & sum & -0.0219356 & -0.021667 & -0.0174941 & -0.0155 & & & 0154646 & -0.0154641 \\
\hline & sum/linear & 0.227133 & 0.22654 & 0.210673 & 0.2003 & & & 199675 & 0.199675 \\
\hline$\widetilde{u^{1}}$ & linear & -0.0402346 & -0.040387 & -0.0432225 & -0.0446 & & & 0447131 & -0.0447136 \\
\hline & quadratic & 0.0335948 & 0.0337304 & 0.0352303 & 0.0357 & & & $\longdiv { 3 5 7 8 5 2 }$ & 0.0357854 \\
\hline & sum & -0.0066398 & -0.0066566 & -0.0079922 & -0.0088 & & & 0089279 & -0.0089282 \\
\hline & sum/linear & 0.165027 & 0.16482 & 0.184908 & $0.1987 !$ & & & 199671 & 0.199675 \\
\hline$\sqrt{k} u^{0,0}$ & linear & 0 & 0 & 0 & 0 & & & 0 & 0 \\
\hline & quadratic & 0 & 0 & 0 & 0 & & & 0 & 0 \\
\hline$\sqrt{k} u^{1,0}$ & linear & 0 & 0 & 0 & 0 & & & 0 & 0 \\
\hline & quadratic & 0 & 0 & 0 & 0 & & & 0 & 0 \\
\hline$\sqrt{k} u^{1,1}$ & linear & 0.0034751 & 0.0001095 & 0.00222367 & 0.000537 & & 0.0 & 0053975 & $1.70688 \times 10^{-6}$ \\
\hline & quadratic & -0.0007686 & -0.00068081 & -0.0000830692 & $-1.01643 \times$ & $0^{-6}$ & -9.95 & $55 \times 10^{-10}$ & $-3.22551 \times 10^{-14}$ \\
\hline$\sqrt{k} u^{1,2}$ & linear & 0 & 0 & 0 & 0 & & & 0 & 0 \\
\hline & quadratic & 0 & 0 & 0 & 0 & & & 0 & 0 \\
\hline
\end{tabular}

Table 7: The values of the equations of motion of $\tilde{t}, \widetilde{u}$ and $\widetilde{u}^{a}$ for $\mathcal{T}_{1}^{[5 / 2,5]}(J=1 / 2)$ 


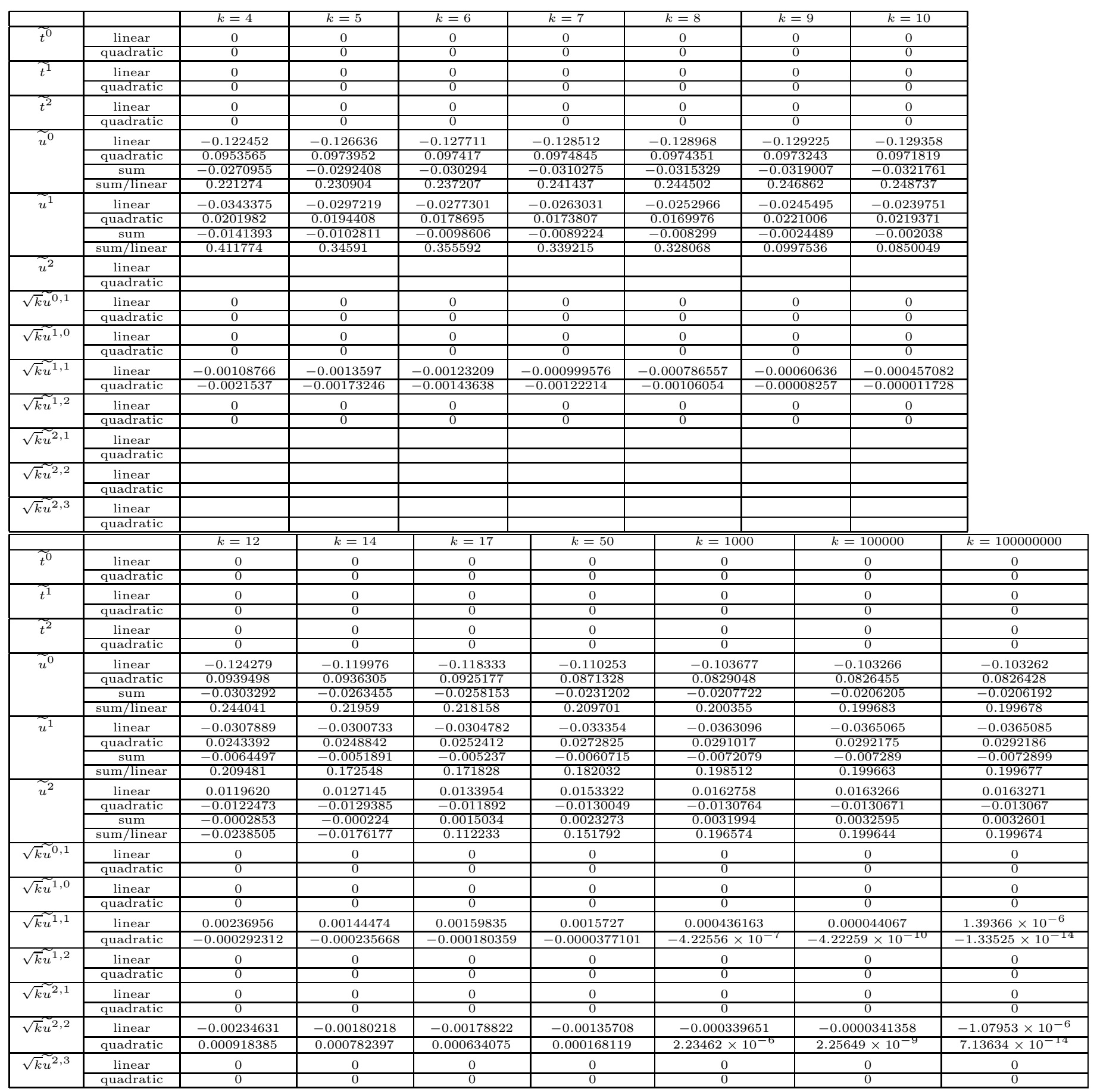

Table 8: The values of the equations of motion of $\widetilde{t}, \widetilde{u}$ and $\widetilde{u}^{a}$ for $\mathcal{T}_{1}^{[5 / 2,5]}(J=1)$ 


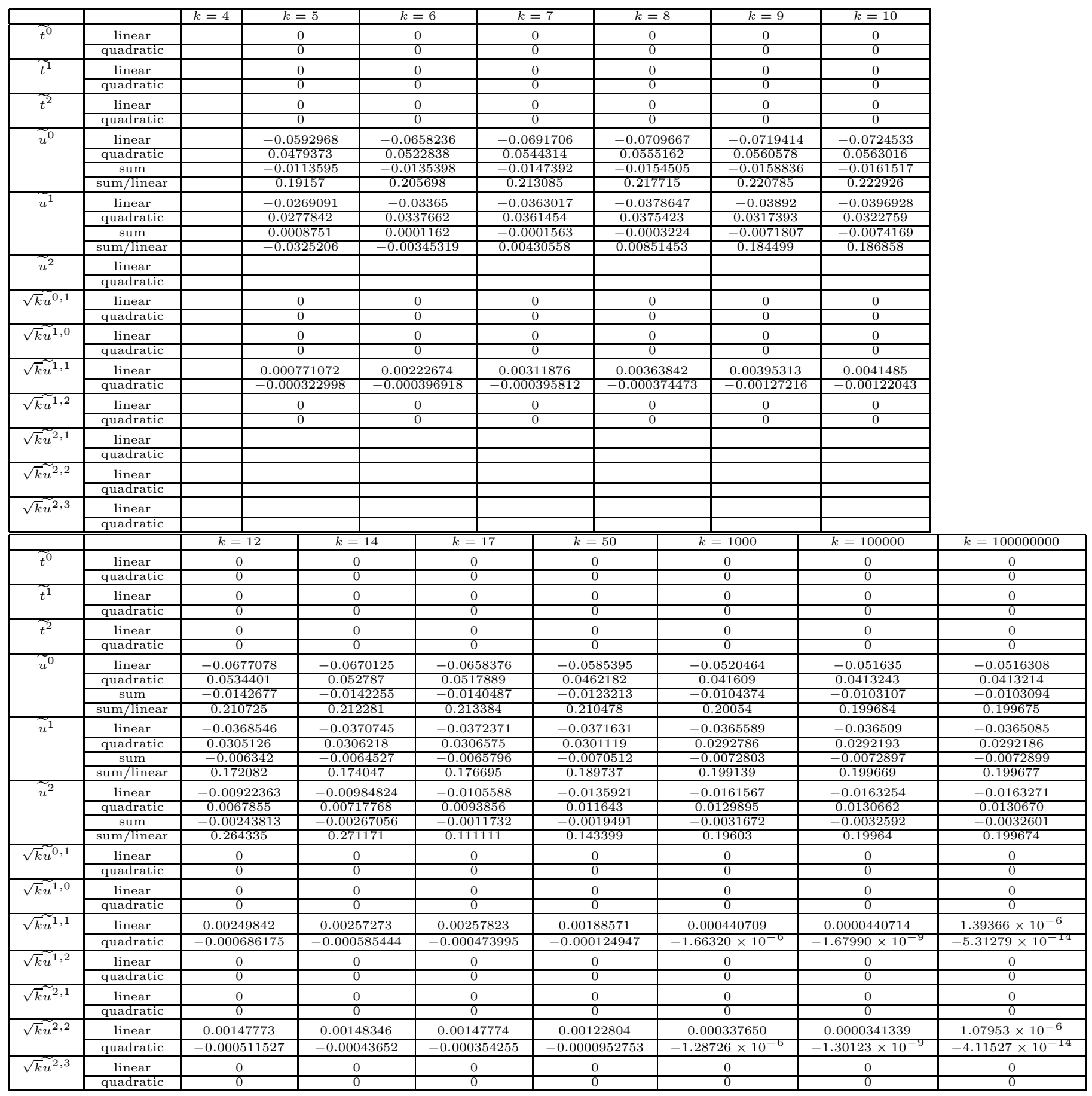

Table 9: The values of the equations of motion of $\widetilde{t}, \widetilde{u}$ and $\widetilde{u}^{a}$ for $\mathcal{T}_{2}^{[5 / 2,5]}(J=1)$ 


\section{References}

[1] A. Sen, "Descent relations among bosonic D-branes", Int. J. Mod. Phys. A14 (1999) 4061 hep-th/9902105

[2] A. Recknagel and V. Schomerus, "Boundary deformation theory and moduli spaces of Dbranes", hep-th/9811237, Nucl. Phys. B545 (1999) 233; C. G. Callan, I. R. Klebanov, A. W. Ludwig and J. M. Maldacena, "Exact solution of a boundary conformal field theory", hep-th/9402113, Nucl. Phys. B422 (1994) 417; J. Polchinski, and L. Thorlacius, "Free fermion representation of a boundary conformal field theory", hep-th/9404008, Phys. Rev. D50 (1994) 622

[3] E. Witten, "Noncommutative geometry and string field theory", Nucl. Phys. B268 (1986) 253

[4] E. Witten, "On background independent open string field theory", Phys. Rev. D46 (5467) 1992, hep-th/9208027 ; E. Witten, "Some computations in background independent offshell string theory", Phys. Rev. D47 (5467) 1992, hep-th/9210065 ; K. Li and E. Witten, "Role of short distance behavior in off-shell open string field theory", Phys. Rev. D48 (853) 1993, hep-th/9303067 ; S. L. Shatashvili, "Comment on the background independent open string theory", Phys. Lett. B311 (83) 1993, hep-th/9303143 ; "On the problems with background independence in string theory", hep-th/9311177;

[5] A. Sen and B. Zwiebach, "Tachyon condensation in string field theory", hep-th/9912249, JHEP 0003 (2000) 002.

[6] V. A. Kostelecký and S. Samuel, "On a Nonperturbative Vacuum for the Open Bosonic String", Nucl. Phys. B336 (1990) 263 ; N. Moeller and W. Taylor, "Level truncation and the tachyon in open bosonic string field theory", hep-th/0002237, Nucl. Phys. B583 (2000) 105

[7] J. A. Harvey, and P. Kraus, "D-branes as unstable lumps in open bosonic string field theory", hep-th/0002117, JHEP 0004 (2000) 012 ; R. de Mello Koch, A. Jevicki, M. Mihailescu and R. Tatar, "Lumps and p-branes in open string field theory", hep-th/0003031, Phys. Lett. B482 (2000) ; N. Moeller,A. Sen and B. Zwiebach, "D-branes as tachyon lumps in string field theory", hep-th/0005036, Phys. Lett. B482 (2000) 249 ; R. de

Mello Koch and J. P. Rodrigues, "Lumps in level truncated open string field theory", 
hep-th/0008053, Phys. Lett. B495 (2000) 237; N. Moeller, "Codimension two lump solutions in string field theory and tachyonic theories", hep-th/0008101

[8] H. Hata and S. Teraguchi, "Test of the Absence of Kinetic Terms around the Tachyon Vacuum in Cubic String Field Theory", hep-th/0101162 ; I. Ellwood and W. Taylor, "Open string field theory without open strings", hep-th/0103085;

[9] L. Rastelli, A. Sen, and B. Zwiebach, "String Field Theory Around the Tachyon Vacuum", hep-th/0012251

[10] L. Rastelli, A. Sen and B. Zwiebach, "Classical solutions in string field theory around the tachyon vacuum", hep-th/0102112 ; B. Feng, Y.-H. He and N. Moeller, "Testing the Uniqueness of the Open Bosonic String Field Theory Vacuum", hep-th/0103103 ; I. Ellwood, B. Feng, Y. -H. He and N. Moeller, "The identity string field and the tachyon vacuum", hep-th/0105024; "Half-strings, projectors, and multiple D-branes in vacuum string field theory", hep-th/0105058 ; D. J. Gross and W. Taylor, "Splitting string field theory I hep-th/0105059 ; T. Kawano and K. Okuyama, "Open string fields as matrices", hep-th/0105129; J. David, "Excitations on wedge states and on the sliver", hep-th/0105184

[11] L. Rastelli, A. Sen and B. Zwiebach, "Boundary CFT construction of D-branes in vacuum string field theory", hep-th/0105168

[12] A. A. Gerasimov and S. L. Shatashvili, "On exact tachyon potential in open string field theory", JHEP 0010 (2000) 034, hep-th/0009103 ; D. Kutasov, M. Mariño and G. Moore, "Some exact results on tachyon condensation in string field theory", JHEP 0010 (2000) 045 , hep-th/0009148

[13] N. Berkovits, "The Tachyon Potential in Open Neveu-Schwarz String Field Theory", hep-th/0001084, JHEP 0004 (2000) 022 ; N. Berkovits, A. Sen and B. Zwiebach, "Tachyon Condensation in Superstring Field Theory", hep-th/0002211, Nucl. Phys. B587 (2000) 147 ; A. Iqbal and A. Naqvi, "Tachyon condensation on a non-BPS Dbrane", hep-th/0004015; P-J. de Smet and J. Raeymaekers, "Level four approximation to the tachyon potential in superstring field theory", hep-th/0003220, JHEP 0005 (2000) 051 ; "The Tachyon Potential in Witten's Superstring Field Theory", hep-th/0004112, JHEP 0008 (2000) 020 ; I. Ya. Aref'eva, D. M. Belov, A. S. Koshelev and P. B. Medvedev, 
"Tachyon condensation in cubic superstring field theory", hep-th/0011117; K. Ohmori, "Tachyonic kink and lump-like solutions in superstring field theory", hep-th/0104230

[14] H. Hata and S. Shinohara, "BRST Invariance of the Non-Perturbative Vacuum in Bosonic Open String Field Theory", hep-th/0009105, JHEP 0009 (2000) 035 ; P. Mukhopadhyay and A. Sen, "Test of Siegel Gauge for the Lump Solution", hep-th/0101014, JHEP 0102 (2001) 017

[15] D. Kutasov, M. Mariño and G. Moore, "Remarks on tachyon condensation in superstring field theory", hep-th/0010108; S. Moriyama and S. Nakamura, "Descent Relation of Tachyon Condensation from Boundary String Field Theory", Phys. Lett. B506 (2001) 16, hep-th/0011002 ; M. Mariño, "On the BV formulation of boundary superstring field theory", hep-th/0103089 ; V. Niarchos and N. Prezas, "Boundary Superstring Field Theory", hep-th/0103102 ; T. Takayanagi, S. Terashima and T. Uesugi, "Brane-Antibrane Action from Boundary String Field Theory", JHEP 0103 (2001) 019, hep-th/0012210 ; P. Kraus and F. Larsen, "Boundary String Field Theory of the $D \bar{D}$ System", hep-th/0012198; S. Frolov, "On off-shell structure of open string sigma model", hep-th/0104042 ; O. Andreev, "More about partition function of open bosonic string in background fields and string theory effective action", hep-th/0104061 ; K. S. Viswanathan and Y. Yang, "Tachyon condensation and background independent superstring field theory", hep-th/0104099 ; M. Alishahiha, "One-loop correction of the tachyon action in boundary superstring field theory", hep-th/0104164 ; S. Nakamura, "Closed string tachyon condensation and on-shell effective action of open string tachyons", hep-th/0105054 ; K. Bardakci and A. Konechny, "Tachyon condensation in boundary string field theory at one loop", hep-th/0105098 ; B. Craps, P. Kraus and F. Larsen, "Loop corrected tachyon condensation", hep-th/0105227

[16] K. Ohmori, "A Review on Tachyon Condensation in Open String Field Theories", hep-th/0102085

[17] A. Sen, "Universality of the tachyon potential", hep-th/9911116, JHEP 9912 (1999) 027

[18] A. Yu. Alekseev, A. Recknagel and V. Schomerus, "Non-commutative World-volume Geometries: Branes on SU(2) and Fuzzy Spheres", hep-th/9908040, JHEP 9909 (1999) 023

[19] A. Yu. Alekseev, A. Recknagel and V. Schomerus, "Brane Dynamics in Background Fluxes and Non-commutative Geometry", hep-th/0003187, JHEP 0005 (2000) 010 
[20] Y. Hikida, M. Nozaki, and T. Takayanagi, "Tachyon Condensation on Fuzzy Sphere and Noncommutative Solitons", hep-th/0008023

[21] C. Bachas, M. Douglas and C. Schweigert, "Flux stabilization of D-branes", hep-th/0003037, JHEP 0005 (2000) 048

[22] A. Yu. Alekseev and V. Schomerus, "D-brane in the WZW model", hep-th/9812193, Phys. Rev. D60 (1999) 061901

[23] J. L. Cardy, "Boundary conditions, fusion rules and Verlinde formula", Nucl. Phys. B324 (1984) 581

[24] N. Ishibashi, "The boundary and crosscap states in conformal field theories", Mod. Phys. Lett. A4 (1989) 251 ; N. Ishibashi and T. Onogi, "Conformal field theories on surfaces with boundaries and crosscaps", Mod. Phys. Lett. A4 (1989) 161

[25] G. Felder, J. Fröhlich, J. Fuchs and C. Schweigert, "The geometry of WZW branes", hep-th/9909030, J. Geom. Phys. 34 (2000) 162

[26] I. Runkel, "Boundary structure constants for the A-series Virasoro minimal models", hep-th/9811178, Nucl. Phys. B549 (1999) 563

[27] A. Sen, "Open string field theory in nontrivial background field: Gauge invariant action", Nucl. Phys. B334 (350) 1990 ; "Open string field theory in nontrivial background field. 2. Feynman rules and four point amplitudes", Nucl. Phys. B334 (395) 1990 ; "Open string field theory in nontrivial background field. 3. N point amplitude", Nucl. Phys. B335 (435) 1990

[28] L. Rastelli and B. Zwiebach, "Tachyon potentials, star products and universality", hep-th/0006240

[29] J. Hoppe, "Diffeomorphism groups, quantization and $S U(\infty)$ ", Int. J. Mod. Phys. A4 (1989) 5235

[30] R. Gopakumar, S. Minwalla and A. Strominger, "Noncommutative solitons", hep-th/0003160, JHEP 0005 (2000) 020 ; K. Dasgupta, S. Mukhi and G. Rajesh, “Noncommutative tachyons", hep-th/0005006, JHEP 0006 (2000) 022 ; J. A. Harvey, P. Kraus, F. Larsen and E. J. Martinec, "D-branes and strings as non-commutative solitons", hep-th/0005031, JHEP 0007 (2000) 042 
[31] E. Witten, "Noncommutative tachyons and string field theory", hep-th/0006071

[32] L. Cornalba and R. Schiappa, "Nonassociative star product deformations for D-brane worldvolumes in curved backgrounds", hep-th/0101219

[33] L. Alvarez-Gaumé, C. Gomez and G. Sierra, "Quantum group interpretation of some conformal field theories", Phys. Lett. B220 (1989) 142 